\title{
Recent Developments in Multidimensional Multirate Systems
}

\author{
Tsuhan Chen, Student Member, IEEE, and P. P. Vaidyanathan, Fellow, IEEE
}

\begin{abstract}
Multidimensional (MD) multirate systems, which find applications in the coding and compression of image and video data, have recently attracted much attention. The basic building blocks in an MD multirate system are the decimation matrix $M$, the expansion matrix $L$, and MD digital filters. With $D$ denoting the number of dimensions, $M$ and $L$ are $D \times D$ nonsingular integer matrices. When these matrices are diagonal, most of the one-dimensional (1-D) multirate results can be extended automatically, using separable approaches (i.e., separable operations in each dimension). Separable approaches are commonly used in practice due to their low complexity in implementation. However, nonseparable operations, with respect to nondiagonal decimation and expansion matrices, often provide more flexibility and better performance. Several applications, such as the conversion between progressive and interlaced video signals, actually require the use of nonseparable operations. For the nonseparable case, extensions of 1-D results to the MD case are nontrivial. Some of these extensions, e.g., polyphase decomposition and maximally decimated perfect reconstruction systems, have already been successfully accomplished by some authors. However, there exist several 1-D results in multirate processing for which the MD extensions are even more difficult. In this paper, we will introduce some recent developments in these extensions. Some important results are: the design of nonseparable MD decimation / interpolation filters derived from 1-D filters, the generalized pseudocirculant property of alias-free maximally decimated filter banks, the commutativity of MD decimators and expanders, and applications in the efficient polyphase implementation of MD rational decimation systems. We will also introduce several other results of theoretical importance.
\end{abstract}

\section{INTRODUCTION}

$\mathrm{F}_{\mathrm{n}}$ OR the one-dimensional (1-D) case, multirate techniques allow the sampling rate in a system to vary from point to point. The basic building blocks are decimators and expanders (also referred to as downsamplers and upsamplers, respectively) [1]-[3]. A typical application of 1-D multirate systems is the subband coding of speech [4], [5] and music [6]. For a recent review of 1-D multirate signal processing, see [3].

Recently, these multirate ideas have been extended to two- and higher-dimensional systems by a number of

Manuscript received December 24, 1991; revised September 11, 1992 and December 8,1992 . This work was supported in part by the National Science Foundation under Grants MIP 8604456 and MIP 8919196 and by matching funds from Tektronix, Inc., Hughes Aircraft Co., and Rockwell International. Paper was recommended by Associate Editor Yrjö Neuvo.

The authors are with the Department of Electrical Engineering, California Institute of Technology, Pasadena, CA 9:125.

IEEE Log Number 9207260. authors [7]-[43]. Multidimensional (MD) multirate systems find applications in the coding and compression of image and video data [8], [10], [12], [20], [30], [35], in sampling format conversions between various video standards [7], [9], high-definition television (HDTV) systems [21], [24], [44], and so on. Central to these systems is the idea of lattices [45], [46], which is closely related to the concepts of MD generalized sampling [47], decimation, and expansion.

An excellent review of MD multirate systems, including key notations and concepts, is given in [26]. Appendix I also provides a summary of these. The key building blocks in MD multirate systems are the decimation matrix $\mathbf{M}$ and the expansion matrix $\mathbf{L}$. With $D$ denoting the number of dimensions, these are $D \times D$ nonsingular integer matrices. When these matrices are diagonal, most of the 1-D results can be automatically extended by performing operations in each dimension separately. However, for the nondiagonal case, these extensions are nontrivial and require more complicated notations and matrix operations. Some of these extensions, e.g., polyphase decomposition and maximally decimated perfect-reconstruction (PR) systems, have already been successfully made by some authors [8], [11], [13]-[15], [18], [23], [26]. A summary of these results can be found in [40, ch. 12]. In particular, [19] and [37] used the McClellan transform to derive MD analysis/synthesis filter banks starting from 1-D linearphase filter banks. Also, [34] extended the relation between multirate filter banks and the discrete wavelet transform to the MD case. However, there still exist several 1-D results in multirate processing for which the multidimensional extensions are even more difficult. In this paper, we will introduce some recent developments in these extensions.

\section{A. Advantages of Nonseparable Operations}

In practice, separable operations are mostly used because of their low implementation complexity. However, nonseparable operations with respect to nondiagonal decimation/expansion matrices are also very important from both theoretical and practical points of view. Nonseparable operations, which include separable operations as a special case, offer more flexibility and better performance and are required in some applications.

As an example, consider the subband coding scheme, where we want to separate a signal into a number of subbands, with each subband corresponding to signal 
components in a certain frequency band. These subband signals are then decimated and quantized. Consider the case where we want to separate a two-dimensional (2-D) image into two parts, the "low"-frequency band and the "high"-frequency band. Suppose only separable operations (separable filtering) are allowed. We can split the signal into two bands with respect to either the horizontal frequency $\omega_{0}$ or the vertical frequency $\omega_{1}$, as shown in Fig. 1(a) and (b). However, if nonseparable operations (nonseparable filters) are used, we can split the image as in Fig. 1(c), where the center square represents the lowfrequency band and the rest represents the high-frequency band. We see that the low-frequency band in Fig. 1(c) is more desirable for this purpose, while those in Fig. 1(a) and (b) are elongated either in $\omega_{0}$ or $\omega_{1}$. This shows the flexibility offered by nonseparable operations. To get more "regular" subband supports (i.e., supports unchanged when $\omega_{0}$ and $\omega_{1}$ are interchanged) when only separable filtering is allowed, we need to use the four-band splitting shown in Fig. 1(d).

For some applications, e.g., multiresolution signal decomposition [48], the frequency splitting is performed repeatedly on the low-frequency band, such that at each step the "resolution" of the resulting low-band signal is decreased by some factor. For example, if we start from Fig. 1(c) (nonseparable) and repeatedly split the low-band signal using the same splitting, we get the frequency splitting in Fig. 2(a). On the other hand, if we start from Fig. 1(a) and (b) (separable), we get the frequency splitting in Fig. 3(a). Although both nonseparable and separable approaches provide a resolution reduction factor of one half at each step, we see that Fig. 3(a) is less desirable because the subband supports are not as regular as those in Fig. 2(a). Of course, we can start from the separable four-band splitting in Fig. 1(d) to get more regular subband supports, but in this case the resolution reduction factor at each step is one quarter, instead of one half.

Nonseparable operations give better performance, too. Consider the application of subband coding again. With nonseparable operations, we can split an image in the frequency domain into nine subband signals (Fig. 2(a)). Since these subband signals are bandlimited, they can be decimated (see Section IX for more details) to keep the total number of samples unchanged. We then quantize these decimated signals according to their energy. (In practice, perceptual properties of the human visual system are also taken into account.) For example, since the energy of most real-world images tends to concentrate in the low-frequency region, we usually assign a larger number of bits to a lower frequency band. By doing this, we reduce the data rate of images. These signals are then recombined to reconstruct the original image. Fig. 2(b) shows the reconstructed image of Lena, which has been quantized to $0.2021 \mathrm{bit} / \mathrm{pixel}$. On the other hand, by using only separable filtering, we can obtain the splitting as in Fig. 3(a). For this case, the reconstructed image of Lena (quantized with the same bit rate, $0.2021 \mathrm{bit} / \mathrm{pixel}$ ) is shown in Fig. 3(b). We can see the better performance

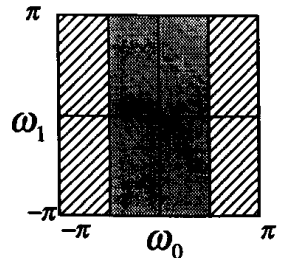

(a)

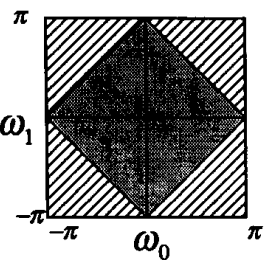

(c)

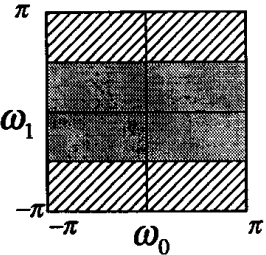

(b)

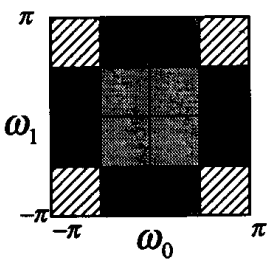

(d)
Fig. 1. Various splitting of 2-D signals: (a) and (b) are associated with separable operations, (c) is nonseparable, and (d) is separable four-band splitting.

obtained by using nonseparable operations in this example.

Remark: The purpose of the above example is only to compare the performance of separable operations and nonseparable operations. By using more complicated coding schemes on the subband signals, e.g. vector quantization, adaptive bit allocation, etc., coded images with higher quality and lower bit rate can be obtained [24], [30], [38]. However, those are not the main points of this example.

For some applications, nonseparable operations are required. The conversion between progressive and interlaced video signals is one example [9], [21]. Progressive signals are obtained by sampling video signals on a rectangular lattice in the time-spatial(vertical) domain, as shown in Fig. 4(a). On the other hand, interlaced signals are obtained by sampling on a nonseparable lattice shown in Fig. 4(b), which is also called the quincunx lattice. The conversion between these two kinds of signals can be achieved by expansion, filtering, and decimation. All of these require nonseparable operations. Another example is directional subband coding, where nondiagonal decimators and nonseparable filters are used to extract image components in different directions [35].

\section{B. Notations}

Capital and lowercase boldfaced letters denote matrices and vectors, respectively. The symbol $\mathbf{I}_{k}$ denotes the $k \times k$ identity matrix (with subscript often omitted). The notations $\mathbf{A}^{T}, \mathbf{A}^{-1}$, and $\mathbf{A}^{-T}$ denote the transpose, the inverse, and the inverse transpose of $\mathbf{A}$, respectively. The row and column indices typically begin at zero. With $D$ denoting the number of dimensions, $\mathbf{n}=\left[\begin{array}{llll}n_{0} & n_{1} & \cdots & n_{D-1}\end{array}\right]^{T}$ is the "time"-domain index of MD discrete signals. For example, $x(\mathbf{n})$ in the 2-D case represents an image. Note that all $n_{i}$ 's are integers. The symbol $\mathscr{N}$ denotes the set of all $D \times 1$ integer vectors, so that $\mathbf{n} \in \mathscr{N}$. The real vector $\boldsymbol{\omega}=\left[\begin{array}{llll}\omega_{0} & \omega_{1} & \cdots & \omega_{D-1}\end{array}\right]^{T}$ is the frequency-domain variable 


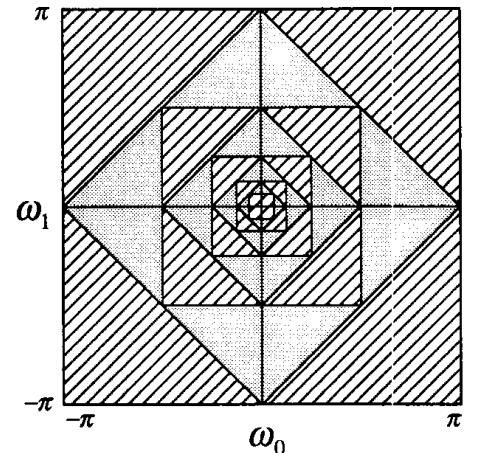

(a)

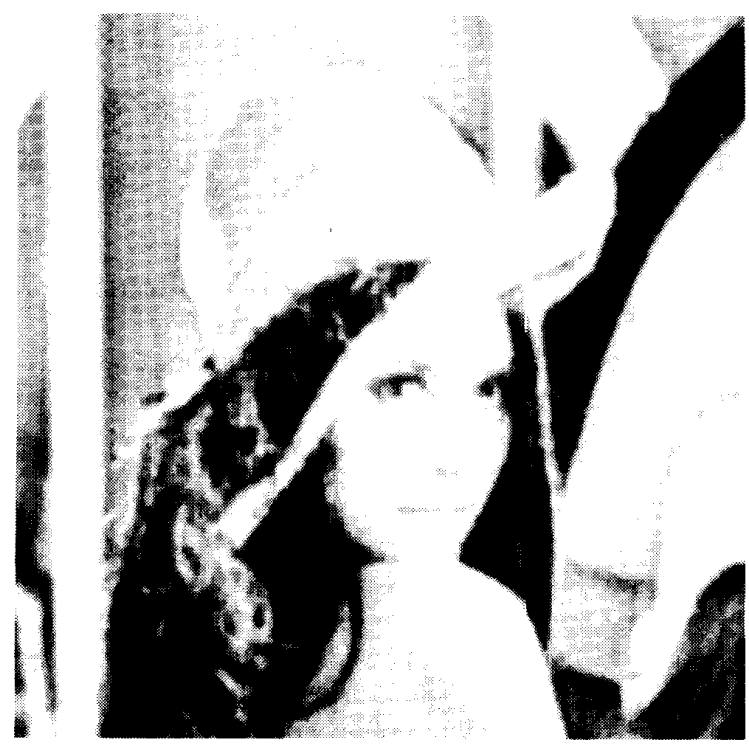

(b)

Fig. 2. Nonseparable subband coding of the Lena image. (a) Frequency splitting. (b) The reconstructed image at $0.2021 \mathrm{bit} / \mathrm{pixel}$.

of MD signals. For example, $X_{F}(\boldsymbol{\omega})$ represents the Fourier transform of $x(\mathbf{n})$ and is defined as

$$
X_{F}(\boldsymbol{\omega})=\sum_{\mathbf{n} \in \mathscr{N}} x(\mathbf{n}) e^{-j \boldsymbol{\omega}^{T} \mathbf{n}}
$$

if the summation converges. By definition, the Fourier transform of an MD signal has periodicity matrix $2 \pi I$ (an MD function $f(\mathbf{x})$ is said to be periodic with periodicity matrix $\mathbf{P}$ if $f(\mathbf{x}+\mathbf{P k})=f(\mathbf{x}), \forall \mathbf{k} \in \mathscr{N})$. The inverse Fourier transform is given by

$$
x(\mathbf{n})=\frac{1}{(2 \pi)^{D}} \int_{\boldsymbol{\omega} \in[-\pi, \pi)^{D}} X_{F}(\boldsymbol{\omega}) e^{j \boldsymbol{\omega}^{T} \mathbf{n}} d \boldsymbol{\omega}
$$

where $[a, b)^{D}$ denotes the set of $D \times 1$ real vectors $\mathbf{x}$ with components $x_{i}$ in the range $a \leq x_{i}<b$. The complex vector $\mathbf{z}=\left[\begin{array}{llll}z_{0} & z_{1} & \cdots & z_{D-1}\end{array}\right]^{T}$ is the variable of the $\mathbf{z}$ transform of MD signals. For example, the $\mathbf{z}$ transform of

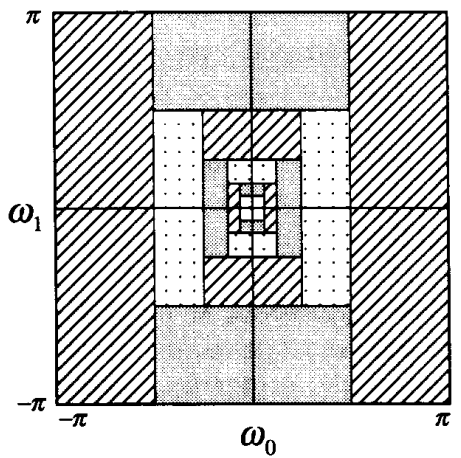

(a)

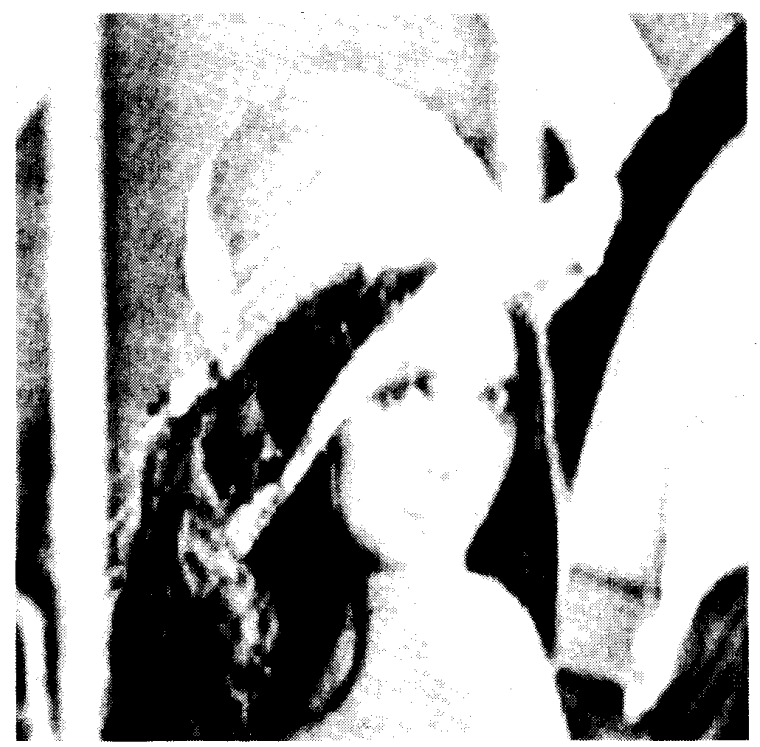

(b)

Fig. 3. Separable subband coding of the Lena image. (a) Frequency splitting. (b) The reconstructed image at $0.2021 \mathrm{bit} / \mathrm{pixel}$.

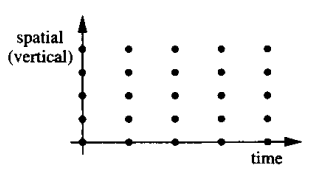

(a)

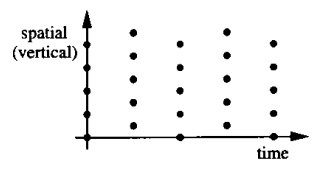

(b)
Fig. 4. Sampling lattices in the time-spatial domain for (a) progressive signals and (b) interlaced signals.

$x(\mathbf{n})$, where it converges, is given by

$$
X(\mathbf{z})=\sum_{\mathbf{n} \in \mathscr{N}} x(\mathbf{n}) \mathbf{z}^{-\mathbf{n}} .
$$

A vector raised to a vector power, as in $z^{-n}$ above, gives a scalar defined as

$$
\mathbf{z}^{\mathbf{n}} \triangleq z_{0}^{n_{0}} \quad z_{1}^{n_{1}} \quad \cdots \quad z_{D-1}^{n_{D-1}} \cdot
$$

Note that the subscript $F$ is used to distinguish the Fourier transform from the $\mathrm{z}$ transform. It is clear that 
$X_{F}(\omega)$ can be obtained by evaluating $X(\mathbf{z})$ at $z_{i}=e^{j \omega_{i}}$ for $i=0, \cdots, D-1$, if it exists.

\section{Basic Building Blocks of MD Multirate Systems}

Decimation: The M-fold decimated version of $x(\mathbf{n})$ is defined as $y(\mathbf{n})=x(\mathbf{M n})$, where $\mathbf{M}$ is a nonsingular integer matrix called the decimation matrix. In the frequency domain, the relation is

$$
Y_{F}(\boldsymbol{\omega})=\frac{1}{J(\mathbf{M})} \sum_{\mathbf{k} \in \mathcal{N}\left(\mathbf{M}^{T}\right)} X_{F}\left(\mathbf{M}^{-r}(\boldsymbol{\omega}-2 \pi \mathbf{k})\right)
$$

where $\mathscr{N}\left(\mathbf{M}^{T}\right)$ is the set of all integer vectors of the form $\mathbf{M}^{T} \mathbf{x}, \mathbf{x} \in[0,1)^{D}$. Also, $J(\mathbf{M})$ denotes $|\operatorname{det} \mathbf{M}|$ (absolute determinant of $\mathbf{M}$ ), which is also equal to the number of elements in $\mathscr{N}\left(\mathbf{M}^{T}\right)$ or $\mathscr{N}(\mathbf{M})$. The $\mathbf{z}$ domain relation of decimation requires more involved notations [26]. Fortunately, for the theoretic derivations of MD multirate results, the frequency-domain relation shown above is usually enough.

Expansion: For a nonsingular integer matrix $\mathbf{L}$, the $\mathbf{L}$ fold expanded version of $x(\mathbf{n})$ is defined as

$$
y(\mathbf{n})= \begin{cases}x\left(\mathbf{L}^{-1} \mathbf{n}\right), & \mathbf{n} \in \operatorname{LAT}(\mathbf{L}) \\ 0, & \text { otherwise }\end{cases}
$$

In the above equation, $L A T(\mathbf{L})$ (the lattice generated by $\mathbf{L}$ [9], [45], [46]) denotes the set of all vectors of the form Lm, m $\in \mathscr{N}$. Clearly, the condition $\mathbf{n} \in L A T(\mathbf{L})$ above is equivalent to $\mathbf{L}^{-1} \mathbf{n} \in \mathscr{N}$. The matrix $\mathbf{L}$ is called the expansion matrix. The corresponding $\mathrm{z}$ domain relation of expansion is

$$
Y(\mathbf{z})=X\left(\mathbf{z}^{\mathbf{L}}\right) .
$$

The notation of a vector raised to a matrix power, as in $z^{\mathbf{L}}$ above, is a $D \times 1$ vector defined as

$$
\mathbf{z}^{\mathbf{P}} \triangleq\left[\begin{array}{llll}
\mathbf{z}^{\mathbf{p}_{0}} & \mathbf{z}^{\mathbf{P}_{1}} & \cdots & \mathbf{z}^{\mathbf{p}_{D-1}}
\end{array}\right]^{T}
$$

where $\mathbf{p}_{i}$ is the $i$ th column of $\mathbf{P}$. In the frequency domain, the relation becomes

$$
Y_{F}(\boldsymbol{\omega})=X_{F}\left(\mathbf{L}^{T} \boldsymbol{\omega}\right) .
$$

Remark: In this paper, unless specified otherwise, $\mathbf{M}$ and $\mathbf{L}$ always denote $D \times D$ nonsingular integer matrices, with the above meanings.

\section{Scope and Outline}

The purpose of this paper is to review some of the fundamental and present some of the recent results in a comprehensive manner. Some of results reported here are new (Sections III, IX, X) while some others have appeared in previous papers (Sections II, IV-VIII).

1) MD Multirate Filters Derived from 1-D Filters (Section II): For the 1-D case, the input to a decimator is usually prefiltered by a so-called "decimation filter" to avoid aliasing. On the other hand, the expander is usually followed by an "interpolation filter" to suppress the image components due to the expander. These are shown in Fig. 5. For $\boldsymbol{M}$-fold decimation/expansion, the decimation/in-

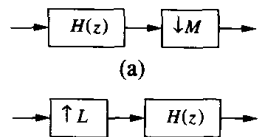

(b)

Fig. 5. One-dimensional (a) decimation system and (b) interpolation system.

terpolation filter typically has passband in the range $\omega \in$ $[-\pi / M, \pi / M)$ [3]. For MD decimation and expansion such filters are also necessary, as indicated in Fig. 6. With M denoting the decimation/expansion matrix, these filters typically have passband in the region

$$
\boldsymbol{\omega}=\pi \mathbf{M}^{-T} \mathbf{x} \quad \mathbf{x} \in[-1,1)^{D} .
$$

In the vector space of $\boldsymbol{\omega}$, such region forms a parallelepiped. In the 2-D case, this region becomes a parallelogram defined by

$$
\begin{aligned}
& -\pi \leq M_{00} \omega_{0}+M_{10} \omega_{1}<\pi \\
& -\pi \leq M_{01} \omega_{0}+M_{11} \omega_{1}<\pi
\end{aligned}
$$

where $M_{i j}$ 's are the elements of the $2 \times 2$ matrix $\mathbf{M}$ (as in [11], [13], [22], [35]). Fig. 7 shows this region for the case

$$
\mathbf{M}=\left[\begin{array}{rr}
1 & -1 \\
1 & 2
\end{array}\right]
$$

Clearly, when $\mathbf{M}$ is not diagonal, these filters are not separable.

Both the design and implementation of nonseparable filters are more complex than those of separable filters [49]. In fact, the complexity grows exponentially with the number of dimensions $D$. Some authors have proposed efficient techniques for the design of some 2-D special filters, e.g., fan filters, diamond-shaped filters, and directional filters, by starting from 1-D prototype filters [11], [13], [16], [22], [35], [50]. Recently, a general method that works for an arbitrary number of dimensions and arbitrary $\mathbf{M}$ has been presented in [25] and [41]. With this method, every filter having a parallelepiped-shaped passband region can be obtained by first designing an appropriate 1-D prototype filter and then performing a simple transformation. MD filter analysis/synthesis filter banks can be designed, too. Hence, the design as well as implementation complexity only grows linearly with the number of dimensions. In Section II, we will outline this method.

2) MD Maximally Decimated Filter Banks (Section III): Fig. 8(a) shows a 1-D analysis/synthesis filter-bank system. Since the number of channels is equal to the decimation ratio $M$, this is also called the maximally decimated filter bank. Using polyphase decomposition and Noble identities [3], we can redraw this system as in Fig. 8(b). The elements in $\mathbf{E}(z)$ and $\mathbf{R}(z)$, denoted as $E_{l, i}(z)$ and $R_{i, l}(z)$, are the polyphase components of the analysis and 


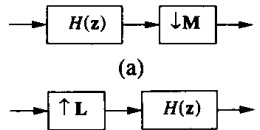

(b)

Fig. 6. MD (a) decimation system and (b) interpolation system.

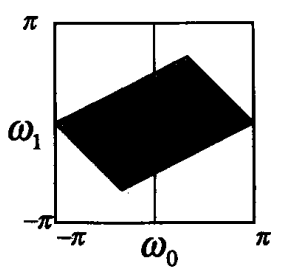

Fig. 7. Typical passband of decimation/interpolation filters.

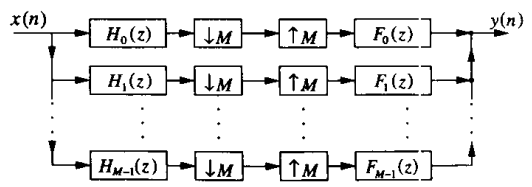

(a)

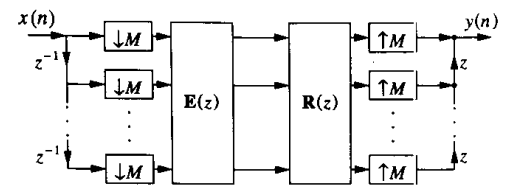

(b)

Fig. 8. One-dimensional maximally decimated filter bank.

synthesis filters, respectively. More specifically

$H_{l}(z)=\sum_{i=0}^{M-1} z^{-i} E_{l, i}\left(z^{M}\right), \quad l=0, \cdots, M-1$

(Type 1 polyphase)

and

$F_{l}(z)=\sum_{i=0}^{M-1} z^{i} R_{i, l}\left(z^{M}\right), \quad l=0, \cdots, M-1$

(Type 2 polyphase).

Define $\mathbf{P}(z)=\mathbf{R}(z) \mathbf{E}(z)$. It is shown in [51] that the necessary and sufficient condition for the system in Fig. 8 to be free from aliasing is that $\mathbf{P}(z)$ has the pseudocirculant property, i.e., $\mathbf{P}(z)$ has the form

$$
\mathbf{P}(z)=\left[\begin{array}{cccc}
P_{0}(z) & z P_{M-1}(z) & \cdots & z P_{1}(z) \\
P_{1}(z) & P_{0}(z) & \cdots & z P_{2}(z) \\
\vdots & \vdots & \ddots & \vdots \\
P_{M-1}(z) & P_{M-2}(z) & \cdots & P_{0}(z)
\end{array}\right]
$$

When $\mathbf{P}(z)$ is the identity matrix $\mathbf{I}$, it can be verified that $y(n)=x(n)$. We say that the filter-bank system achieves PR if the output is same as the input up to a scale factor and/or a delay, i.e., $y(n)=c_{0} x\left(n-n_{0}\right)$ for some integer $n_{0}$. The condition $\mathbf{P}(z)=\mathbf{I}$, although commonly used, is not necessary for PR. The necessary and sufficient condition for $\mathrm{PR}$ is that $\mathbf{P}(z)$ should have the form

$$
\mathbf{P}(z)=c_{0} z^{-m_{0}}\left[\begin{array}{cc}
\mathbf{0} & z \mathbf{I}_{r} \\
\mathbf{I}_{(M-r)} & \mathbf{0}
\end{array}\right]
$$

for some $c_{0} \neq 0$, some integer $m_{0}$, and some $0 \leq r \leq M$ -1 [52].

The concept of MD maximally decimated filter bank has been proposed by many authors [11], [13]-[15], [18], [23], [26]. Using MD polyphase decomposition, we can obtain the corresponding $\mathbf{P}(\mathbf{z})$ of an MD maximally decimated filter bank. When the decimation/expansion matrix $\mathbf{M}$ is diagonal, it has been shown that the filter-bank system is alias-free if and only if the corresponding $\mathbf{P}(\mathbf{z})$ is multidimensional pseudocirculant [15]. For the nondiagonal case, conditions for freedom from aliasing have been given in [14], [18], [26] in terms of eigenvectors and eigenvalues of $\mathbf{P}(\mathbf{z})$. In Section III, we shall derive the so-called generalized pseudocirculant property of $\mathbf{P}(\mathbf{z})$ and show that this property is necessary and sufficient for a filter-bank system to be alias-free. This adds more insight about the structure of $\mathbf{P}(\mathbf{z})$ of alias-free filter-bank systems.

3) Smith Form of Integer Matrix and Applications (Section $I V)$ : Most of the difficulties in extending 1-D multirate results to the MD case come from the fact that the decimation/expansion matrices may not be diagonal. It is well-known that any integer matrix can be diagonalized by using the Smith form [53]. Therefore, the Smith form is very useful for diagonalizing and thus simplifies many MD multirate problems [28], [29], [32] as we will explain in Section IV.

4) Least Common Multiples of Integer Matrices and the Applications (Sections V-VIII): The greatest common divisor ( $\mathrm{gcd})$, least common multiple $(\mathrm{lcm})$, and coprimeness of integers are well-known and very often appear in the contexts of 1-D multirate systems. Here are some examples:

1) An $M$-fold decimator and an $L$-fold expander can be interchanged (i.e., the system in Fig. 9(a) is equivalent to the system in Fig. 9(b)) if and only if $M$ and $L$ are relatively prime (coprime) [3].

2) Rational decimation systems, as shown in Fig. 10(a), play a very important role in audio systems. This scheme permits us to alter the sampling rate of a sequence by a rational number $M / L$. The filter $H(z)$ is used to suppress image components generated by the $L$-fold expander and to eliminate aliasing due to the $M$-fold decimator. Using polyphase technique, we can implement Fig. 10(a) more efficiently, as in Fig. 10(b) (for the case $M=3$ and. $L=2$ ). It turns out that we can improve the efficiency even further by using the technique introduced in [54]. We shall refer to this technique as the rational polyphase implementation (RPI). Fig. 11 


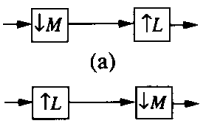

(b)

Fig. 9. Interchange of a 1-D decimator and a 1-D expander.

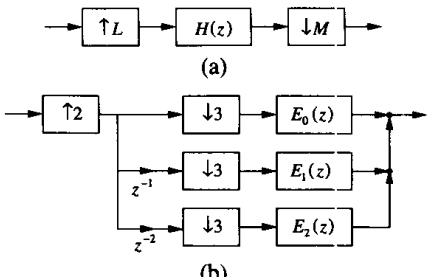

Fig. 10. (a) One-dimensional rational decimction system and (b) the polyphase implementaticn.

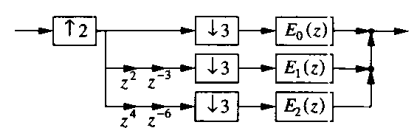

(a)

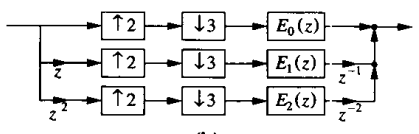

(b)

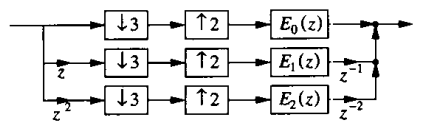

(c)

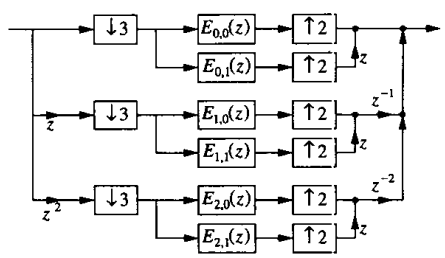

(d)

Fig. 11. Successive redrawing of polyphase irnplementations of a 1-D rational decimation system.

shows the development of the RPI technique by successively redrawing the rational decimation circuit. Starting from Fig. 10(b), we replace $z^{-1}$ by $z^{2} z^{-3}$ and $z^{-2}$ by $z^{4} z^{-6}$, so we get Fig. 11(a). With the help of Noble identities [3], Fig. 11(a) can be redrawn as Fig. 11(b). Next, we can interchange the expanders and decimators when $M$ and $L$ are coprime and obtain Fig. 11(c). Finally, we can perform Type 2 polyphase decomposition on $E_{i}(z)$ 's with respect to $L$, and get Fig. 11(d). In summary, Fig. 11(d) is equivalent to Fig. 10(a), but now each arithmetic operation is performed at its lowest rate. Note that the RPI technique works if and only if $M$ and $L$ are coprime.
3) It is easily verified that the system in Fig. 12 is a PR system, i.e., $\hat{x}(n)=x(n)$. This system is fundamental to many 1-D maximally decimated PR filter banks [3]. We can generalize this by replacing every $z$ with $z^{L}$ and obtain the so-called delay-chain system in Fig. 13. This system is a PR system if and only if $L$ and $M$ are coprime [55, lemma A.2]. The case where $L \neq 1$ is required in some applications where pairs of analysis filters are constrained by symmetry conditions [55].

4) When a periodic signal $x(n)$ with period $L$ is decimated by a factor of $M$ to obtain $y(n)=x(M n)$, the period of $y(n)$ is $L / \operatorname{gcd}(M, L)=\operatorname{lcm}(M, L) / M$. Also, when a cyclo-wide-sense-stationary (CWSS) random sequence $x(n)$ with period $L$ is decimated by $M$, the resulting sequence is still CWSS and has period $L / \operatorname{gcd}(M, L)=\operatorname{lcm}(M, L) / M[56]$.

In Sections V-VIII, we shall extend all these ideas to the MD case. To do so, we need the concepts of $\mathrm{gcd}, \mathrm{lcm}$, and coprimeness for matrices. For polynomial matrices, the greatest common left/right divisors (gcld/gcrd), right/left coprimeness, matrix-fraction descriptions, the Smith form, the Smith-McMillan form, and so on, are well-known in the system-theory area [57], [58], [59]. In fact, these properties can also be applied to the integermatrix case. ${ }^{1}$ In Section V, we will introduce the concepts of least common right multiples (lcrm) and least common left multiples (1clm) and several properties of them. By using all these tools, we can extend the above-mentioned four issues to the MD case. The following results, which appeared in [31], [36], [39], can be obtained:

1) An $\mathbf{M}$-fold decimator and an $\mathbf{L}$-fold expander are interchangeable if and only if $\mathbf{M}$ and $\mathbf{L}$ commute (i.e., $\mathbf{M L}=\mathbf{L M}$ ) and are coprime (to be explained in Section VI). In general, we have to distinguish right coprimeness and left coprimeness for the matrix case. However, we will show that when $\mathbf{M L}=\mathbf{L M}$, right coprimeness and left coprimeness are equivalent. This interchangeability problem was first addressed in [27] for upper triangular $\mathbf{M}$ and $\mathbf{L}$ in the 2-D case.

2) An MD decimation system with rational decimation ratio (in this case, a matrix) $\mathbf{H}=\mathbf{L}^{-1} \mathbf{M}$ finds applications in the conversion of images or video data between different sampling standards. MD rational decimation systems can be implemented efficiently by using the so-called MD RPI technique whenever $\mathbf{M}$ and $\mathbf{L}$ are left coprime (Section VI).

3) An MD delay-chain system (which is an extension of Fig. 13, to be defined later) is a PR system if $\mathbf{L M}$ is an $1 \mathrm{crm}$ of $\mathbf{M}$ and $\mathbf{L}$ (Section VII). However, the necessary condition for this is still an open problem. One potential application of MD delay-chain sys-

\footnotetext{
${ }^{1}$ More generally, these properties can be applied to matrices with elements from a so-called "principle ideal domain" (pid) [46], [59], [60], [61]. The set of integers and the set of polynomials with coefficients belonging to a field are two examples of pid's.
} 


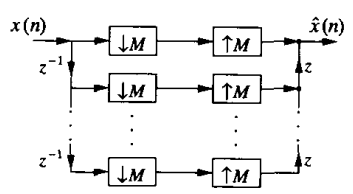

Fig. 12. A 1-D perfect reconstruction system.

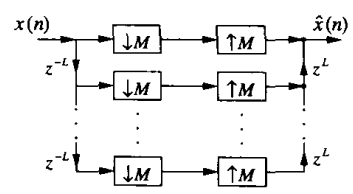

Fig. 13. One-dimensional delay-chain system.

tems is to design MD filter banks where the analysis and synthesis filters have a certain symmetry. The research on this is under progress.

4) When an MD periodic signal $x(\mathbf{n})$ with periodicity matrix $\mathbf{L}$ is decimated by a factor of $\mathbf{M}$, the periodicity matrix of the resulting sequence is $\mathbf{M}^{-1}$ $\operatorname{lcrm}(\mathbf{M}, \mathbf{L})$, where $\operatorname{lcrm}(\mathbf{M}, \mathbf{L})$ denotes an $\operatorname{lcrm}$ of $\mathbf{M}$ and $\mathbf{L}$ (Section VIII). A similar result holds for the random-signal case.

5) Conditions for Alias-Free Decimation (Section IX): For the 1-D case, a decimation filter $H_{F}(\omega)$ preceding an $M$-fold decimator to avoid aliasing typically has the passband in the range $\omega \in[\pi / M, \pi / M)$. However, this is not the only choice. For example, consider the filters in Fig. 14. It can be verified that any of these can precede a three-fold decimator to avoid aliasing. All of these filters have one common property, i.e., the support (the region where the frequency response is nonzero) of any of these filters does not overlap under the "modulo- $(2 \pi / M)$ " operation (in this case $M=3$ ). The arrows in Fig. 14 show the "modulo- $(2 \pi / 3)$ " operation graphically. Basically, every component in the support is translated by some proper multiple of $2 \pi / M$ so that all components are "folded" into the region $[0,2 \pi / M)$. It has been shown in [56] that "no overlap under modulo- $(2 \pi / M)$ operation" is both necessary and sufficient for a decimation. filter to eliminate aliasing due to an $M$-fold decimator. In Section IX, we will extend this result to the MD case. We will show that the necessary and sufficient condition for alias-free $\mathbf{M}$-fold decimation is that the support of the decimation filter $H_{F}(\omega)$ does not overlap under "modulo- $2 \pi \mathbf{M}^{-T}$ ", operation (to be defined later).

6) Multistage Design of Multirate Systems (Section X): In the $1-D$ case, multistage design has been used to implement decimation systems with large decirnation ratios [1, sec. 5.1]. Fig. 15(a) shows a two-stage decimation system with overall decimation ratio $M=M_{1} M_{2}$. Using Noble identities [3], this can be redrawn as in Fig. 15(b), where the filter $H(z)=H_{1}(z) H_{2}\left(z^{M_{1}}\right)$ belongs :o the so-called interpolated finite-impulse-response (IFIR) filters [62]. IFIR structure has the advantage that narrowband FIR
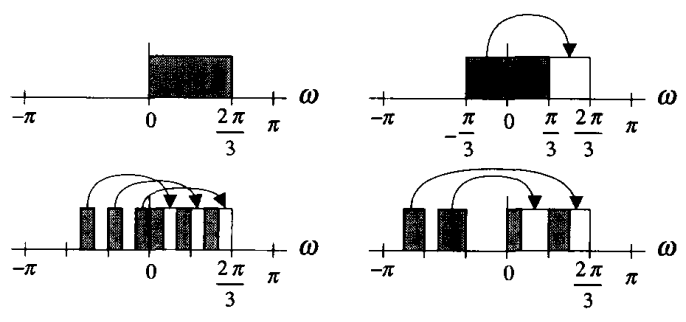

Fig. 14. Several possible decimation filters for the threefold decimation.

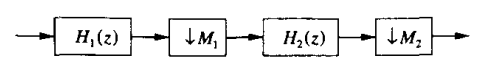

(a)

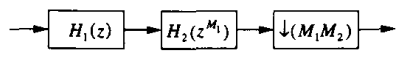

(b)

Fig. 15. One-dimensional multistage decimation system.

filters can be designed efficiently and implemented with significant savings in the number of arithmetic operations, compared with conventional direct methods. The same multistage idea can be extended to the MD case if we decompose the decimation matrix as $\mathbf{M}=\mathbf{M}_{1} \mathbf{M}_{2}$ properly. In Section $X$, we will mention the details and point out some subtle issues that do not have a 1-D counterpart.

This paper concludes with some remarks on open problems in this area. In Appendix I, a list of important notations and basic concepts of MD multirate signal processing is provided for quick reference.

\section{MD FILTERS DERIVED FROM 1-D FILTERS}

In MD multirate signal processing, filters with parallelepiped-shaped passband support are commonly used as decimation/interpolation filters. Since transfer functions with such supports are in general nonseparable, both the design and implementation of these filters have complexity that grows exponentially with the number of dimensions $D$ [48]. Some authors have proposed efficient techniques for the design of some special cases of 2-D filters by starting from 1-D prototype filters [11], [13], [16], [22], [35], [50]. In this section, we will outline a general method with which all filters with passband in the region

$$
\boldsymbol{\omega}=\pi \mathbf{M}^{-T} \mathbf{x}, \quad \mathbf{x} \in[-1,1)^{D}
$$

can be designed starting from an appropriate 1-D prototype filter [25], [41]. So, the design as well as the implementation complexity of these filters only grows linearly with the number of dimensions. This method works for arbitrary number of dimensions and arbitrary $\mathbf{M}$. Further advantages and properties of this method will be summarized at the end of this section.

We shall use the notation $S P D(V)$ (symmetric parallelepiped generated by $\mathbf{V}$ ) to denote the set of all real 
vectors of the form $\mathbf{V} \mathbf{x}$, for $\mathbf{x} \in[-1,1)^{D}$. Clearly, we can then rewrite (17) as $\omega \in \operatorname{SPD}\left(\pi \mathbf{M}^{-T}\right)$.

Remark: By definition, the Fourier transform of an MD signal has periodicity matrix $2 \pi$ I. To be more precise, we should use

$$
\operatorname{SPD}\left(\pi \mathbf{M}^{-T}\right)+2 \pi \mathbf{m}, \quad \mathbf{m} \in \mathscr{N}
$$

to express the passband support of these parallelepipedshaped filters. That is, the region $\operatorname{SPD}\left(\pi \mathbf{M}^{-T}\right)$ should be repeated every $2 \pi$ in each dimension to give the whole support of the passband. However, for simplicity, we do not show the term $2 \pi \mathbf{m}$ explicitly.

\section{A. Impulse Response of an Ideal Low-Pass Filter}

To explain our method, we first derive the impulse response $h(\mathbf{n})$ of an ideal low-pass filter $H_{F}(\boldsymbol{\omega})$ with $\operatorname{SPD}\left(\pi \mathbf{M}^{-T}\right)$ as the passband. This expression will reveal a fundamental relation to 1-D low-pass filters and enable us to design $H_{F}(\omega)$ starting from a 1-D prototype. Let

$$
H_{F}(\boldsymbol{\omega})= \begin{cases}1, & \text { if } \boldsymbol{\omega} \in \operatorname{SPD}\left(\pi \mathbf{M}^{-T}\right) \\ 0, & \text { otherwise. }\end{cases}
$$

Using the inverse-Fourier-transform relation in (2), we can obtain

$$
\begin{aligned}
h(\mathbf{n}) & =\frac{1}{(2 \pi)^{D}} \int_{\boldsymbol{\omega} \in S P D\left(\pi \mathbf{M}^{-T}\right)} e^{j \boldsymbol{\omega}^{T} \mathbf{n}} d \boldsymbol{\omega} \\
& =\frac{1}{2^{D} J(\mathbf{M})} \int_{\mathbf{x} \in[-1,1)^{D}} e^{j \pi \mathbf{x}^{T} \mathbf{M}^{-1} \mathbf{n}} d \mathbf{x}, \\
& =\frac{1}{2^{D} J(\mathbf{M})} \int_{x \in[-1,1)^{D}} e^{j \pi \mathbf{x}^{T} \mathbf{m}} d \mathbf{x}, \quad\left(\mathbf{\omega}=\pi \mathbf{M}^{-T} \mathbf{x}\right) \\
& \left.=\frac{1}{2^{D} J(\mathbf{M})} \prod_{i=0}^{D-1} \int_{x_{i}=-1}^{1} e^{j \pi m_{i} x_{i}} d x_{i} \mathbf{n}\right) \\
& =\frac{1}{J(\mathbf{M})} \prod_{i=0}^{D-1} \frac{\sin \left(\pi m_{i}\right)}{\pi m_{i}}
\end{aligned}
$$

where $m_{i}$ are the components of the $L \times 1$ vector

$$
\mathbf{m}=\mathbf{M}^{-1} \mathbf{n}=\frac{\hat{\mathbf{M}} \mathbf{n}}{J(\mathbf{M})}
$$

with $\hat{\mathbf{M}} \triangleq J(\mathbf{M}) \cdot \mathbf{M}^{-1}= \pm$ [adjugate (adjoint) of $\left.\mathbf{M}\right]$. Since the adjugate of an integer matrix is an integer matrix, $\hat{\mathbf{M}}$ must be an integer matrix.

\section{B. Relationship to 1-D Filters}

Consider a 1-D ideal filter with frequency response $P_{F}(\omega)$ as shown in Fig. 16. Its impulse response is given by

$$
p(n)=\frac{\sin \left(\frac{\pi n}{J(\mathbf{M})}\right)}{\pi n} .
$$

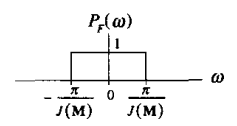

Fig. 16. Frequency response of an ideal 1-D low-pass filter.

Starting from this prototype filter $P_{F}(\omega)$, suppose we define the MD filter

$$
H_{F}^{(s)}(\boldsymbol{\omega})=P F\left(\omega_{0}\right) P_{F}\left(\omega_{1}\right) \cdots P_{F}\left(\omega_{D-1}\right) .
$$

This is a separable low-pass filter, with passband support $S P D(\pi \mathbf{I} / J(\mathbf{M}))$. Its impulse response, also separable, is

$$
h^{(s)}(\mathbf{n})=p\left(n_{0}\right) p\left(n_{1}\right) \cdots p\left(n_{D-1}\right)=\prod_{i=0}^{D-1} \frac{\sin \left(\frac{\pi n_{i}}{J(\mathbf{M})}\right)}{\pi n_{i}}
$$

Now consider the quantity $h^{(s)}(\hat{\mathbf{M}} \mathbf{n})$, which is the $\hat{\mathbf{M}}$-fold decimated version of $h^{(s)}(\mathbf{n})$. Since $\hat{\mathbf{M}} \mathbf{n}=J(\mathbf{M}) \mathbf{M}^{-1} \mathbf{n}=$ $J(\mathbf{M}) \mathbf{m}$, we get

$$
\begin{aligned}
h^{(s)}(\hat{\mathbf{M}} \mathbf{n}) & =h^{(s)}(J(\mathbf{M}) \mathbf{m})=\sum_{i=0}^{D-1} \frac{\sin \left(\pi m_{i}\right)}{\pi J(\mathbf{M}) m_{i}} \\
& =\frac{1}{J(\mathbf{M})^{D}} \prod_{i=0}^{D-1} \frac{\sin \left(\pi m_{i}\right)}{\pi m_{i}} .
\end{aligned}
$$

Comparing (25) with (20), we obtain the following very simple relation between $h(\mathbf{n})$ and $h^{(s)}(\hat{\mathbf{M}} \mathbf{n})$

$$
h(\mathbf{n})=c h^{(s)}(\hat{\mathbf{M}} \mathbf{n})
$$

where $c=[J(\mathbf{M})]^{D-1}=J(\hat{\mathbf{M}})$. In other words, $h(\mathbf{n})$ is obtained simply by $\hat{\mathbf{M}}$-fold decimation of the separable sequence $h^{(s)}(\mathbf{n})$, followed by scaling with $c$ !

\section{Design Procedure and Example}

Motivated by the above conclusion, we can design an MD filter with the passband support $\operatorname{SPD}\left(\pi \mathbf{M}^{-T}\right)$ as follows:

Step 1) Design a 1-D low-pass prototype filter $P_{F}(\omega)$, which approximates the response of Fig. 16.

Step 2) Construct the separable MD filter $h^{(s)}(\mathbf{n})$ from $p(n)$, as in (24).

Step 3) Decimate $h^{(s)}(\mathbf{n})$ by $\hat{\mathbf{M}}$ and scale by $c$ to obtain $h(\mathbf{n})$, as in (26).

We now present a design example for the case

$$
\mathbf{M}=\left[\begin{array}{rr}
1 & -1 \\
1 & 2
\end{array}\right]
$$

We use linear programming [63, sec. 3.19] to design the prototype filter $P_{F}(\omega)$, an FIR filter with length $N=59$, passband ripple $\delta_{1}=0.01994$, and stopband ripple $\delta_{2}=$ $0.00888(-41.03 \mathrm{~dB})$, shown in Fig. 17(a). Following the steps described above, we obtain $H_{F}^{(s)}(\boldsymbol{\omega})$ and $H_{F}(\boldsymbol{\omega})$, as shown in Fig. 17(b) and (c). The resulting $H_{F}(\omega)$ has passband ripple $\delta_{1}^{\prime}=0.03931$ and stopband ripple $\delta_{2}^{\prime}=$ $0.01778(-35.00 \mathrm{~dB})$. 


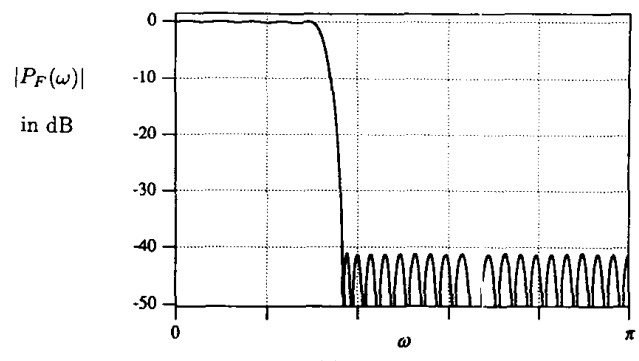

(a)
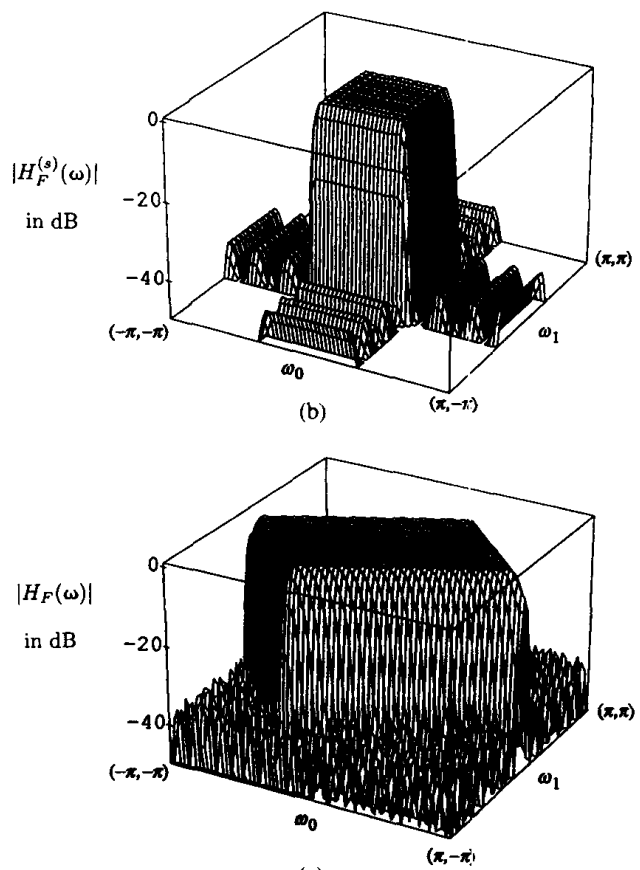

(c)

Fig. 17. Frequency response of the design exarnple. (a) $P_{F}(\omega)$, (b) $H_{F}^{(s)}(\boldsymbol{\omega}),(\mathrm{c}) H_{F}(\boldsymbol{\omega})$.

\section{Further Comments About the Method}

More details about this method can be found in [25] and [41]. Some of them are summarized here:

1) Suppose the prototype filter $P_{F}(\omega)$ has passband ripple $\delta_{1}$ and stopband ripple $\delta_{2}$. It can be shown that the peak passband and stopband ripples of the resulting filter $H_{F}(\omega)$ are upper bounded by

$$
\hat{\delta}_{1}=(J(\hat{\mathbf{M}})-1) \delta_{2}+D \delta_{1}, \quad \hat{\delta}_{2}=J(\hat{\mathbf{M}}) \delta_{2}
$$

2) An MD filter having purely real frequency response is called a zero-phase filter [49, p. 113]. Zero-phase filters introduce no phase distortion, which is important in many MD applications, especially in image processing. On the other hand, an MD filter with impulse response $h(\mathbf{n})$ satisfying $h(\mathbf{M n})=0$ for $\mathbf{n} \neq \mathbf{0}$ is also called a Nyquist filter or Mth-band filter [16]. When used as interpolation filters in $\mathbf{M}$-fold interpolation, Mth-band Nyquist filters have the advantage that the values of existing samples can be preserved (i.e., no intersymbol interference). It can be proved that both the zero-phase property and Nyquist (Mth-band) property are preserved under the proposed transformation. More specifically, if we want $H_{F}(\omega)$ to be a zero-phase filter, we can achieve this by designing the 1-D filter $P_{F}(\omega)$ to have zero phase. Also, if we want $H_{F}(\boldsymbol{\omega})$ to be an Mth-band Nyquist filter, we can achieve this by designing the $1-\mathrm{D}$ filter $P_{F}(\omega)$ to be a $J(\mathbf{M})$ th-band Nyquist filter. Moreover, stability is also preserved. That is, if we begin with a stable IIR prototype filter $P_{F}(\omega)$, the resulting $H_{F}(\omega)$ is also guaranteed to be stable.

3) Because of the separability of $H_{F}^{(s)}(\omega)$, the proposed method is efficient not only in the design, but also in the implementation. Suppose the prototype $p(n)$ has $N$ coefficients. Therefore, $h^{(s)}(\mathbf{n})$ has $N^{D}$ coefficients and $h(\mathbf{n})$ has approximately $N^{D} / J(\hat{\mathbf{M}})$ coefficients. Hence, to implement $h(\mathbf{n})$ directly requires approximately $N^{D} / J(\hat{\mathbf{M}})$ arithmetic operations per computed output pixel (OPP's). Instead of direct implementation, we can schematically represent $H_{F}(\omega)$ in terms of $H_{F}^{(s)}(\omega)$ as in Fig. 18. Because $H_{F}^{(s)}(\omega)$ is separable, this implementation requires only $J(\hat{\mathbf{M}}) \cdot D N$ OPP's, where the factor $J(\hat{\mathbf{M}})$ is due to the $\hat{\mathbf{M}}$-fold expander. Hence we have reduced the complexity from $\mathscr{O}\left(N^{D}\right)$ to $\mathscr{O}(N)$.

4) In addition to filter design, the design and implementation of MD filter banks can also be simplified by using the proposed approach. More specifically, we can start from a 1-D $J(\mathbf{M})$-channel uniform discrete Fourier transform (DFT) filter bank to derive an MD uniform-DFT filter bank [41]. As an example, a 2-D two-channel PR filter bank, where the filters have more than $35 \mathrm{~dB}$ stopband attenuation using only two multiplications per input pixel, can be found in [41]

5) Note that $\operatorname{SPD}\left(\pi \mathbf{M}^{-T}\right)$ does not represent all parallelepipeds, because elements of $\mathbf{M}$ are restricted to be integers. To represent all possible parallelepipeds, we need to use $\operatorname{SPD}\left(\pi \mathbf{H}^{-T}\right)$ where $\mathbf{H}$ is a matrix with rational elements. (The irrational case can be approximated by rational matrices.) To design a filter having passband support $\operatorname{SPD}\left(\pi \mathbf{H}^{-T}\right)$, we proceed as follows. Given the nonsingular rational matrix $\mathbf{H}$, we can write it as $\mathbf{H}=$ $\mathbf{L}^{-1} \mathbf{M}$, the so-called matrix fraction description (MFD) [58], [59]. We first design $h(\mathbf{n})$ with passband support $\operatorname{SPD}\left(\pi \mathbf{M}^{-T}\right)$ as before. Then, we decimate $h(\mathbf{n})$ by $\mathbf{L}$ to obtain $g(\mathbf{n})$. It is shown in [41] that the passband support of $g(\mathbf{n})$ is exactly $\operatorname{SPD}\left(\pi \mathbf{H}^{-T}\right)$.

6) In (23), if we choose different 1-D filters in different dimensions, more flexibility in the transition bandwidth and stopband attenuation of the resulting filter can be obtained.

\section{MD MaXimally Decimated Filter Banks}

An MD analysis/synthesis filter bank with decimation/expansion matrix $\mathbf{M}$ is shown in Fig. 19(a). When the number of channels is equal to $J(\mathbf{M})$, this is called a maximally decimated filter bank. To analyze this system, we use the MD polyphase decomposition [23], [26] 


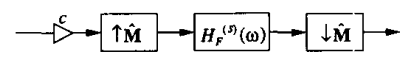

Fig. 18. Efficient implementation of an MD filter.

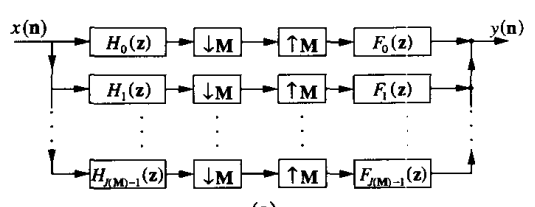

(a)

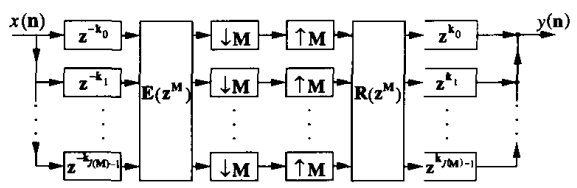

(b)

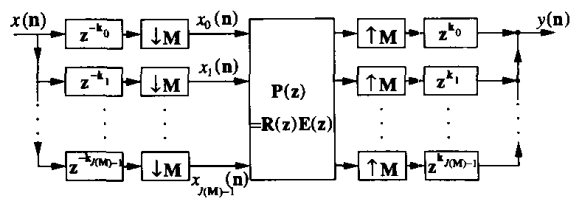

(c)

Fig. 19. MD maximally decimated filter bank.

defined as follows:

Polyphase Decomposition: The polyphase components of $x(\mathbf{n})$ with respect to a given $\mathbf{M}$ are defined as

$$
e_{i}(\mathbf{n})=x\left(\mathbf{M n}+\mathbf{k}_{i}\right) \quad(\text { Type } 1)
$$

or

$$
r_{i}(\mathbf{n})=x\left(\mathbf{M n}-\mathbf{k}_{i}\right) \quad(\text { Type } 2)
$$

where $\mathbf{k}_{i} \in \mathscr{N}(\mathbf{M})$. The vector $\mathbf{k}_{i}$ can take on $J(\mathbf{M})$ different values, which are ordered as $\mathbf{k}_{0}, \mathbf{k}_{1}, \cdots, \mathbf{k}_{J(\mathbf{M})-1}$. In the $\mathbf{z}$-domain, the polyphase decomposition of $X(\mathbf{z})$ can be expressed as

$$
X(\mathbf{z})=\sum_{\mathbf{k}_{i} \in \mathcal{N}(\mathbf{M})} \mathbf{z}^{-\mathbf{k}_{i}} E_{i}\left(\mathbf{z}^{\mathbf{M}}\right) \quad \text { (Type 1) }
$$

or

$$
X(\mathbf{z})=\sum_{\mathbf{k}_{i} \in \mathscr{M}(\mathbf{M})} \mathbf{z}^{\mathbf{k}_{i}} R_{i}\left(\mathbf{z}^{\mathbf{M}}\right) . \quad(\text { Type 2) }
$$

In the frequency domain, these become

$$
X_{F}(\boldsymbol{\omega})=\sum_{\mathbf{k}_{i} \in \mathcal{N}(\mathbf{M})} e^{-j \boldsymbol{\omega}^{T} \mathbf{k}_{i}} E_{F, i}\left(\mathbf{M}^{T} \boldsymbol{\omega}\right) \quad \text { (Type 1) }
$$

or

$$
X_{F}(\boldsymbol{\omega})=\sum_{\mathbf{k}_{i} \in \mathcal{N}(\mathbf{M})} e^{j \omega^{T} \mathbf{k}_{i}} R_{F, i}\left(\mathbf{M}^{T} \boldsymbol{\omega}\right) . \quad \text { (Type 2) }
$$

Note that $\mathbf{z}^{\mathbf{k}}$ (or $e^{j \omega^{T} \mathbf{k}}$ ) is a shift operator, which shifts (advances) an MD signal by a vector amount $\mathbf{k}$. Similarly, $\mathbf{z}^{-\mathbf{k}}$ (or $e^{-j \omega^{T} \mathbf{k}}$ ) shifts an MD signal by $-\mathbf{k}$, i.e., "delays" it by $\mathbf{k}$.

Using the polyphase decomposition, we can represent each analysis filter and synthesis filter in the form

$$
H_{l}(\mathbf{z})=\sum_{\mathbf{k}_{i} \in \mathcal{N}(\mathbf{M})} \mathbf{z}^{-\mathbf{k}_{i}} E_{l, i}\left(\mathbf{z}^{\mathbf{M}}\right), \quad l=0, \cdots, J(\mathbf{M})-1
$$

and

$$
F_{l}(\mathbf{z})=\sum_{\mathbf{k}_{i} \in \mathcal{N}(\mathbf{M})} \mathbf{z}^{\mathbf{k}_{i}} R_{i, l}\left(\mathbf{z}^{\mathbf{M}}\right), \quad l=0, \cdots, J(\mathbf{M})-1 .
$$

Note that the vectors in $\mathscr{N}(\mathbf{M})$ are ordered as $\mathbf{k}_{0}, \mathbf{k}_{1}, \cdots, \mathbf{k}_{J(M)-1}$ and $\mathbf{k}_{0}$ is usually chosen to be the zero vector $\mathbf{0}$. Then, we can redraw this system as in Fig. 19(b). The $J(\mathbf{M}) \times J(\mathbf{M})$ matrices $\mathbf{E}(\mathbf{z})$ and $\mathbf{R}(\mathbf{z})$ with elements $E_{l, i}(\mathbf{z})$ and $R_{i, l}(\mathbf{z})$ are called the polyphase matrices for the analysis bank and the synthesis bank, respectively.

MD Noble identities (shown in Fig. 20) allow us to move the decimators and expanders across $\mathbf{E}\left(\mathbf{z}^{\mathbf{M}}\right)$ and $\mathbf{R}\left(\mathbf{z}^{\mathbf{M}}\right)$, respectively, and obtain Fig. 19(c). Define

$$
\mathbf{P}(\mathbf{z})=\mathbf{R}(\mathbf{z}) \mathbf{E}(\mathbf{z})
$$

The goal is to find the necessary and sufficient conditions on $\mathbf{P}(\mathbf{z})$ such that the system is free from aliasing. When this is true, the filter bank becomes a linear time-invariant (LTI) system with a certain transfer function $T(\mathbf{z})$. After the aliasing has been canceled, we want to find the conditions such that $T(\mathbf{z})=c_{0} \mathbf{z}^{-\mathbf{n}_{0}}$. In this case, $y(\mathbf{n})=$ $c_{0} x\left(\mathbf{n}-\mathbf{n}_{0}\right)$ and we say the system achieves PR.

Remark: It is obvious that when $\mathbf{P}(\mathbf{z})=\mathbf{I}$, the filter bank achieves PR and, in fact, $y(\mathbf{n})=x(\mathbf{n})$.

Recall that in the 1-D case, the maximally decimated filter bank is alias-free if and only if $\mathbf{P}(z)$ is pseudocirculant [51] and achieves PR if and only if $\mathbf{P}(z)$ has the form in (16) [52]. We now extend these ideas to the MD case. Theorem 1 and Corollary 1 give the main results. The reader can scan them at this time. The rest of this section is devoted to their derivation.

Consider the filter-bank system in Fig. 19(c). It can be verified that this is a linear periodic-time-varying system with periodicity matrix $\mathbf{M}$ (denoted as $\left.(L P T V)_{\mathbf{M}}\right)$. That is, with $y(\mathbf{n})$ denoting the output of such a linear system for a certain input $x(\mathbf{n}), y(\mathbf{n}+\mathbf{M k})$ will be the out for $x(\mathbf{n}+$ Mk) for every $\mathbf{k} \in \mathscr{N}$. We also know that the system is alias-free if and only if it is indeed LTI. That is, $y(\mathbf{n}+\mathbf{k})$ is the output for the input $x(\mathbf{n}+\mathbf{k})$ for all $\mathbf{k} \in \mathscr{N}$. Therefore, to check if a $(L P T V)_{\mathrm{M}}$ system is free from aliasing, we only have to check if $y(\mathbf{n}+\mathbf{k})$ is the output for the input $x(\mathbf{n}+\mathbf{k})$ for every nonzero $\mathbf{k} \in \mathscr{N}(\mathbf{M})$.

Let the input to the system be an impulse function at the origin, i.e., $x(\mathbf{n})=\delta(\mathbf{n})$ so $X(\mathbf{z})=1$. Consider the input signals to the transfer matrix $\mathbf{P}(\mathbf{z}): x_{l}(\mathbf{n}), l=$ $0, \cdots, J(M)-1$ as indicated in Fig. 19(c). We obtain

$$
X_{l}(\mathbf{z})= \begin{cases}1, & l=0 \\ 0, & \text { otherwise. }\end{cases}
$$

Then, it is easily verified that the output $Y(\mathbf{z})$ is

$$
Y(\mathbf{z})=\sum_{\mathbf{k}_{j} \in \mathcal{N}(\mathbf{M})} \mathbf{z}^{\mathbf{k}_{j}} P_{j, 0}\left(\mathbf{z}^{\mathbf{M}}\right)
$$




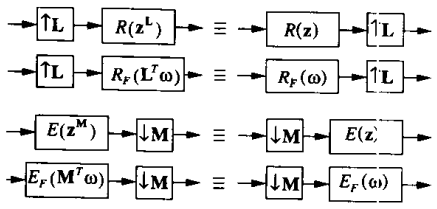

Fig. 20. MD Noble identities.

where $P_{j, i}(\mathbf{z})$ is the $(j, i)$ th element of $\mathbf{P}(\mathbf{z})$. Now, let the input to the system be $x(\mathbf{n})=\delta\left(\mathbf{n}+\mathbf{k}_{i}\right)$ for some $\mathbf{k}_{i}$ in $\mathscr{N}(\mathbf{M})$. For such input, we obtain

$$
X_{l}(\mathbf{z})= \begin{cases}1, & l=i \\ 0, & \text { otherwise } .\end{cases}
$$

For this case, the output $Y^{\prime}(\mathbf{z})$ is

$$
Y^{\prime}(\mathbf{z})=\sum_{\left.\mathbf{k}_{l} \in \mathcal{M} \mathbf{M}\right)} \mathbf{z}^{\mathbf{k}_{l}} P_{l, i}\left(\mathbf{z}^{\mathbf{M}}\right) .
$$

For this system to be LTI, we must have

$$
\begin{gathered}
\underbrace{\mathbf{z}^{\mathbf{k}_{i}} \sum_{\mathbf{k}_{j} \in \mathcal{N}(\mathbf{M})} \mathbf{z}^{\mathbf{k}_{j}} P_{j, 0}\left(\mathbf{z}^{\mathbf{M}}\right)}_{Y(\mathbf{z})}=\underbrace{\sum_{\mathbf{k}_{l} \in \mathcal{N}(\mathbf{M})} \mathbf{z}^{\mathbf{k}_{t}} P_{l, i}\left(\mathbf{z}^{\mathbf{M}}\right)}_{Y^{\prime}(\mathbf{z})} \\
\forall i=1, \cdots, J(\mathbf{M})-1
\end{gathered}
$$

to ensure that the filter-bank system is LTI. The above equation can be written as

$$
\begin{aligned}
\sum_{\mathbf{k}_{j} \in \mathcal{N}(\mathbf{M})} \mathbf{z}^{\mathbf{k}_{i}+\mathbf{k}_{j}} P_{j, 0}\left(\mathbf{z}^{\mathbf{M}}\right)= & \sum_{k_{l} \in \mathcal{N}(\mathbf{M})} \mathbf{z}^{\mathbf{k}_{l}} P_{l, i}\left(\mathbf{z}^{\mathbf{M}}\right), \\
& \forall i=1, \cdots, J(\mathbf{M})-1 .
\end{aligned}
$$

The division theorem [48, sec. 2.4.2] says that every integer vector $\mathbf{n}$ can be uniquely expressed as $\mathbf{n}=\mathbf{M} \mathbf{n}_{0}+$ $\mathbf{k}$ for some $\mathbf{k} \in \mathscr{N}(\mathbf{M})$ and $\mathbf{n}_{0} \in \mathscr{N}$. With modulo notation, the "remainder" $\mathbf{k}$ can be expressed as $\mathbf{k}=\mathbf{n} \bmod \mathbf{M}$, or $\mathbf{k}=((\mathbf{n}))_{\mathbf{M}}$ (please see Appendix II for the computation of $\left.((\mathbf{n}))_{M}\right)$. Therefore, we can express the term $\mathbf{k}_{i}+\mathbf{k}_{j}$ in the left-hand side of (40) as

$$
\mathbf{k}_{i}+\mathbf{k}_{j}=\mathbf{M g}(i, j)+\mathbf{k}_{f(i, j)}
$$

where $\mathbf{g}(i, j) \in \mathscr{N}$ and $\mathbf{k}_{f(i, j)} \in \mathscr{N}(\mathbf{M})$. In other words, $\mathbf{g}(i, j)$ is the "quotient" of $\mathbf{k}_{i}+\mathbf{k}_{j}$ with respect to $\mathbf{M}$ and $\mathbf{k}_{f(i, j)}$ is the "remainder," i.e., $\mathbf{k}_{f(i, j)}=\left(\left(\mathbf{k}_{i}+\mathbf{k}_{j}\right)\right)_{\mathbf{M}}$. Note that both $\mathbf{g}(i, j)$ and $f(i, j)$ are functions of $i$ and $j$ for $i, j=$ $0, \cdots, J(\mathbf{M})-1$. Also note that $\mathbf{g}(i, j)$ and $f(i, j)$ are determined by the matrix $\mathbf{M}$ and the ordering of $\mathbf{k}_{i}$ 's in $\mathscr{M}(\mathbf{M})$.

Using these two functions $\mathbf{g}(i, j)$ and $f(i, j)$, we can rewrite (40) as

$$
\begin{array}{r}
\sum_{\mathbf{k}_{j} \in \mathscr{N}(\mathbf{M})} \mathbf{z}^{\mathbf{k}_{f(i, j)} \mathbf{z}^{\mathbf{M g}(i, j)} P_{j, 0}\left(\mathbf{z}^{\mathbf{M}}\right)}=\sum_{\mathbf{k}_{l} \in \mathscr{N}(\mathbf{M})} \mathbf{z}^{\mathbf{k}_{l}} P_{l, i}\left(\mathbf{z}^{\mathbf{M}}\right), \\
\forall i=1, \cdots, J(\mathbf{M})-1 .
\end{array}
$$

This leads to

$$
\begin{aligned}
\mathbf{z}^{\mathbf{g}(i, j)} P_{j, 0}(\mathbf{z})=P_{f(i, j), i}(\mathbf{z}), \quad & \forall i=1, \cdots, J(\mathbf{M})-1, \\
\forall j & =0, \cdots, J(\mathbf{M})-1 .
\end{aligned}
$$

Polynomial matrices satisfying the above relation will be called generalized-pseudocirculant matrices with respect to $\mathbf{M}$ (for a specific ordering of $\mathbf{k}_{i}$ 's in $\mathscr{N}(\mathbf{M})$ ). Note that for generalized-pseudocirculant matrices, after the first column is known, the other $J(\mathbf{M})-1$ columns are completed determined by (43). In particular, the function $f(i, j)$ decides how to put the elements of the first column in the other $J(\mathbf{M})-1$ columns, and the function $\mathbf{g}(i, j)$ decides the quantity to be multiplied for each element.

For example, let

$$
\mathbf{M}=\left[\begin{array}{rr}
1 & 1 \\
-1 & 2
\end{array}\right]
$$

We order the elements in $\mathscr{N}(\mathbf{M})$ as follows

$$
\mathbf{k}_{0}=\left[\begin{array}{l}
0 \\
0
\end{array}\right], \quad \mathbf{k}_{1}=\left[\begin{array}{l}
1 \\
0
\end{array}\right], \quad \mathbf{k}_{2}=\left[\begin{array}{l}
1 \\
1
\end{array}\right] .
$$

Then, the generalized-pseudocirculant property in (43) implies that $\mathbf{P}(\mathbf{z})$ has the form

$$
\mathbf{P}(\mathbf{z})=\left[\begin{array}{ccc}
P_{0}(\mathbf{z}) & z_{0} z_{1} P_{2}(\mathbf{z}) & z_{0} z_{1} P_{1}(\mathbf{z}) \\
P_{1}(\mathbf{z}) & P_{0}(\mathbf{z}) & z_{1} P_{2}(\mathbf{z}) \\
P_{2}(\mathbf{z}) & z_{0} P_{1}(\mathbf{z}) & P_{0}(\mathbf{z})
\end{array}\right] .
$$

We have proved the following theorem:

Theorem 1: The MD $J(\mathbf{M})$-channel maximally decimated filter bank is free from aliasing if and only if $\mathbf{P}(\mathbf{z})$ is generalized pseudocirculant. More specifically, when $\mathbf{P}(\mathbf{z})$ is generalized pseudocirculant, the filter bank is equivalent to an LTI system with overall transfer function

$$
T(\mathbf{z})=\sum_{\mathbf{k}_{j} \in \mathcal{N}(\mathbf{M})} \mathbf{z}^{\mathbf{k}_{j}} P_{j, 0}\left(\mathbf{z}^{\mathbf{M}}\right) .
$$

Remarks:

1) The relation in (43) reduces to the 1-D pseudocirculant property nicely. For $\mathbf{M}=M$, we order the elements in $\mathscr{N}(M)$ in the conventional way, i.e., $\{0,1, \cdots, M-1\}$, so that

$$
f(i, j)=((i+j))_{M} \quad \text { and } \quad \mathbf{g}(i, j)=g(i, j)=\left\lfloor\frac{i+j}{M}\right\rfloor
$$

where $\lfloor x\rfloor$ denotes the largest integer less than or equal to $x$. Then, (43) becomes

$$
\begin{cases}P_{j, 0}(z)=P_{i+j, i}(z), & j<M-i \\ z P_{j, 0}(z)=P_{i+j-M, i}(z), & j \geq M-i \\ & \forall i=1, \cdots, M-1\end{cases}
$$

which implies that $\mathbf{P}(z)$ has the form in (15).

2) When $\mathbf{M}$ is diagonal, it has been shown that the necessary and sufficient condition for an alias-free filter bank is that $\mathbf{P}(\mathbf{z})$ should be multidimensional pseudocircu lant [15]. When $\mathbf{M}$ is diagonal and the vectors in $\mathscr{N}(\mathbf{M})$ are put in the lexicographic order, the relations in (43) are 
verified to be equivalent to the multidimensional-pseudocirculant property.

After aliasing has been removed, we can consider the conditions for PR. Using (47), we can obtain the following corollary:

Corollary 1: The MD filter bank achieves PR if and only if $\mathbf{P}(\mathbf{z})$ is generalized pseudocirculant and all $P_{j, 0}(\mathbf{z})$ 's are zero except that one $P_{j, 0}(\mathbf{z})$ is equal to a delay. More specifically, if

$$
P_{j, 0}(\mathbf{z})= \begin{cases}c_{0} \mathbf{z}^{\mathbf{m}_{0}}, & j=j_{(1} \\ 0, & \text { otherwise }\end{cases}
$$

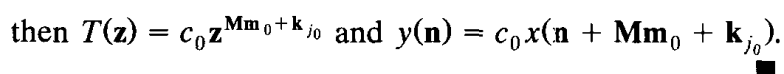

\section{SMITH Form OF INTEGER MATRICES AND APPLICATIONS}

When the decimation/expansion matrices are diagonal, most of the 1-D multirate techniques can be easily extended to the MD case simply by using separable approaches. When these matrices are not diagonal, these extensions are nontrivial. Therefore, the Smith form, which diagonalizes integer matrices, is very important for MD multirate signal processing. The Smith form for integer matrices has been known for over 100 years [53]. Its polynomial version finds many applications in system theory [57]-[59]. The Smith form for integer matrices has also been applied in the context of MD signal processing in [28], [29], [32], and [64]. The Smith form provides many valuable insights and is a useful tool to establish theoretical results in MD multirate signal processing, but this does not solve all design problems.

Smith Form [53]: Any nonsingular integer matrix $\mathbf{M}$ can always be decomposed as $\mathbf{M}=\mathbf{U} \mathbf{\Lambda} \mathbf{V}$, where $\mathbf{U}$ and $\mathbf{V}$ are unimodular integer matrices (matrices with determinant \pm 1 ), and $\boldsymbol{\Lambda}$ is a diagonal matrix with positive-integer elements on the diagonal. Also, the diagonal elements $\lambda_{i}$ 's of $\Lambda$ satisfy $\lambda_{i} \mid \lambda_{i+1}$, i.e., $\lambda_{i}$ is a factor of $\lambda_{i+1}$. Under these conditions, $\boldsymbol{\Lambda}$ is unique for a given $\mathbf{M}$, but $\mathbf{U}$ and $\mathbf{V}$ are not (and are, in general, infinitely many). For example

$$
\underbrace{\left[\begin{array}{rr}
1 & -1 \\
1 & 2
\end{array}\right]}_{\mathbf{M}}=\underbrace{\left[\begin{array}{ll}
1 & 0 \\
1 & 1
\end{array}\right]}_{\mathbf{U}} \underbrace{\left[\begin{array}{ll}
1 & 0 \\
0 & 3
\end{array}\right]}_{\mathbf{\Lambda}} \underbrace{\left[\begin{array}{rr}
1 & -1 \\
0 & 1
\end{array}\right]}_{\mathbf{V}} .
$$

The Smith form brings many MD issues much closer to the separable case. The first observation we make is that an $\mathrm{M}$-fold decimator can be redrawn as in Fig. 21. Since the decimation by a unimodular matrix is only a permutation of samples of $x(\mathbf{n})$ (no actual decimation involved), the $\mathbf{M}$-fold decimator can be interpreted as: 1) a permutation of samples of $x(\mathbf{n})$ by $\mathbf{U}, 2)$ a decimation in the $i$ th dimension by a factor of $\lambda_{i}$ separately in each dimension, and 3) a permutation by $\mathbf{V}$. Another application is that, by factorizing the elements $\lambda_{i}$ 's into their prime factors, we can identify all nontrivial multistage implementations of a given decimator.

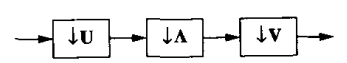

Fig. 21. Smith-form decomposition of an $\mathbf{M}$-fold decimator.

The Smith form can also be applied to the design of decimation filters, as shown in Fig. 22(a). Using Noble identities and the fact that the decimation by unimodular $\mathbf{U}$ is equal to expansion by $\mathbf{U}^{-1}$, the decimation system in Fig. 22(a) can be redrawn as in Fig. 22(b), with $G(\mathbf{z})=$ $H\left(\mathbf{z}^{U^{-1}}\right)$. Because decimation by a unimodular matrix does not introduce aliasing at all, only the $\boldsymbol{\Lambda}$-fold decimator may cause aliasing. For the diagonal $\boldsymbol{\Lambda}$, we can design $G(\mathbf{z})$ to be separable, i.e., $G(\mathbf{z})=G_{0}\left(z_{0}\right) \cdots G_{D-1}\left(z_{D-1}\right)$. We let $G_{i}\left(z_{i}\right)$ have support $\left[-\pi / \lambda_{i}, \pi / \lambda_{i}\right)$ and hence prevent aliasing in the overall system. In this case, the equivalent decimation filter $H(\mathbf{z})$ has support $S P D$ $\left(\pi(\mathbf{U} \Lambda)^{-T}\right)$ rather than $\operatorname{SPD}\left(\pi \mathbf{M}^{-T}\right)$.

As another application, the Smith form gives us a way to characterize all decimation matrices of a given decimation ratio. Let us choose $J(\mathbf{M})=4$ as an example. For the 2-D case, $\Lambda$ has only two possible forms

$$
\Lambda=\left[\begin{array}{ll}
1 & 0 \\
0 & 4
\end{array}\right] \text { and } \boldsymbol{\Lambda}=\left[\begin{array}{ll}
2 & 0 \\
0 & 2
\end{array}\right]
$$

Therefore, all of the 2-D decimation matrices with ratio 4 can be characterized by either one of the above two forms premultiplied and postmultiplied by unimodular matrices.

The Smith-form decomposition also applies to maximally decimated filter-bank systems. Consider the filterbank system in Fig. 19(a). Using $\mathbf{M}=\mathbf{U} \mathbf{\Lambda V}$, we can redraw each branch as in Fig. 23(a). Note that the unimodular matrix $\mathbf{V}$ cancels off. We then use Noble identities to rearrange this as in Fig. 23(b) where

$$
G_{i}(\mathbf{z})=H_{i}\left(\mathbf{z}^{\mathrm{u}^{-1}}\right) \text { and } Q_{i}(\mathbf{z})=F_{i}\left(\mathbf{z}^{\mathbf{u}^{-1}}\right) .
$$

In other words, we have redrawn the original filter-bank system $\mathscr{S}_{\mathbf{M}}$ in terms of a new filter-bank system $\mathscr{S}_{\boldsymbol{\Lambda}}$ as in Fig. 24. Since the decimators and expanders in the system $\mathscr{S}_{\mathrm{A}}$ are diagonal, the theory developed for the 1-D case can now be easily extended to the MD case. The relation between these two systems is summarized as follows:

1) The original system $\mathscr{S}_{\mathrm{M}}$ is alias-free if and only if $\mathscr{S}_{\Lambda}$ is alias-free. In the alias-free case, the overall transfer functions of these two systems, $T_{\mathbf{M}}(\mathbf{z})$ and $T_{\Lambda}(\mathbf{z})$ are related as $T_{\mathbf{M}}(\mathbf{z})=T_{\Lambda}\left(\mathbf{z}^{\mathbf{v}}\right)$. As mentioned earlier, $\mathscr{S}_{\Lambda}$ with diagonal decimators and expanders is alias-free if and only if the corresponding polyphase matrix $\mathbf{P}_{\mathbf{\Lambda}}(\mathbf{z})$ is multidimensional pseudocirculant [15]. This also gives a necessary and sufficient condition for the original system $\mathscr{S}_{\mathrm{M}}$ to be free from aliasing.

2) Under alias-free conditions,

a) $\mathscr{S}_{\mathbf{M}}$ is free from amplitude distortion if and only if $\mathscr{S}_{\mathbf{\Lambda}}$ is.

b) $\mathscr{S}_{\mathrm{M}}$ is free from phase distortion if and only if $\mathscr{S}_{\mathrm{A}}$ is.

c) $\mathscr{S}_{\mathrm{M}}$ achieves PR if and only if $\mathscr{S}_{\Lambda}$ does. 


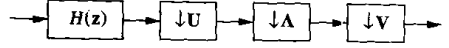

(a)

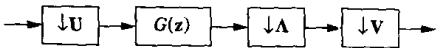

(b)

Fig. 22. Decimation filter with Smith-form decomposition.

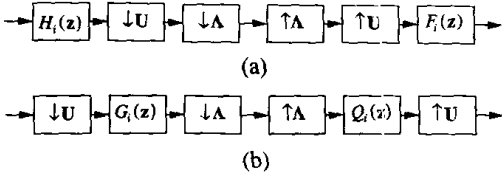

Fig. 23. One branch of an analysis/synthesis filter-bank system

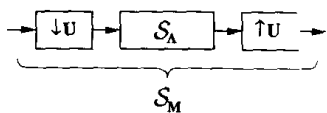

Fig. 24. Equivalent filter-bank system

The Smith form also helps to find the generalized MD discrete-Fourier-transform (DFT) matrix $\mathbf{W}^{(g)}$ for a given M. This matrix, with elements defined as

$$
\begin{aligned}
{\left[\mathbf{W}^{(g)}\right]_{i, j}=e^{-j 2 \pi \mathbf{m}_{i}^{T} \mathbf{M}^{-1} \mathbf{k}_{j},} } & \\
\mathbf{m}_{i} & \in \mathscr{N}\left(\mathbf{M}^{T}\right), \quad \mathbf{k}_{j} \in \mathscr{N}(\mathbf{M})
\end{aligned}
$$

is used in computing the discrete Fotrier transform of MD signals [49, sec. 2.4.1] and in the design and implementation of MD-uniform DTF filter banks [23]. Let $\mathbf{M}=\mathbf{U} \boldsymbol{\Lambda} \mathbf{V}$ and let $\mathbf{W}_{\lambda_{i}}$ be the traditional $\lambda_{i} \times \lambda_{i}$ DFT matrix, i.e., $\left[\mathbf{W}_{\lambda_{i}}\right]_{m, k}=e^{-j 2 \pi m k / \lambda_{i}}$. It can be shown that $\mathbf{W}^{(g)}$ is a Kronecker product of all the $\mathbf{W}_{\lambda_{i}}$ 's, i.e.

$$
\mathbf{W}^{(g)}=\mathbf{P}_{1}\left[\mathbf{W}_{\lambda_{0}} \otimes \mathbf{W}_{\lambda_{1}} \otimes \cdots \otimes \mathbf{W}_{\lambda_{D-1}}\right] \mathbf{P}_{2}
$$

where $\mathbf{P}_{1}$ and $\mathbf{P}_{2}$ are appropriate permutation matrices depending on $\mathbf{U}, \mathbf{V}$, and the ordering of the $\mathbf{k}_{j}$ 's and $\mathbf{m}_{i}$ 's in (54). The Kronecker product of two matrices is defined as

$$
\begin{aligned}
& {\left[\begin{array}{ccc}
a_{0,0} & \cdots & a_{0, J-1} \\
\vdots & & \vdots \\
a_{K-1,0} & \cdots & a_{K-1, J-1}
\end{array}\right] \otimes \mathbf{B} } \\
& \triangleq\left[\begin{array}{ccc}
a_{0,0} \mathbf{B} & \cdots & a_{0, J-1} \mathbf{B} \\
\vdots & & \vdots \\
a_{K-1,0} \mathbf{B} & \cdots & a_{K-1, J-1} \mathbf{B}
\end{array}\right] .
\end{aligned}
$$

The Smith form can also be used to design a computer algorithm for finding all elements in $\mathscr{N}(\mathbf{M})$ [32]. In general, elements in $\mathscr{N}(\mathbf{M})$ can be found by searching through all integer vectors $\mathbf{n}$ and checking if $((\mathbf{n}))_{M}=\mathbf{n}$, until all $J(\mathbf{M})$ elements are found. ${ }^{2}$ For a real nonsingular matrix

\footnotetext{
${ }^{2}$ Since $\mathscr{M}(\mathbf{M})$ forms a group under addition modulo $\mathbf{M}$, the coset decomposition [65, sec. 2.2] of $\mathscr{N}(\mathbf{M})$ helps to expedite such searching.
}

$\mathbf{V}$, we define the fundamental parallelepiped generated by $\mathbf{V}$ (denoted by $F P D(\mathbf{V})$ ) as the set of all real vectors of the form $\mathbf{V x}$, where $\mathbf{x} \in[0,1)^{D}$. The set $\mathscr{N}(\mathbf{M})$ is therefore the set of all integer vectors in $F P D(M)$. Hence we can reduce the searching range for $\mathscr{N}(\mathbf{M})$ to the rectangular that circumscribes $F P D(\mathbf{M})$. For example, Fig. 25 shows such circumscribing rectangular for the $\mathbf{M}$ given in (27). This searching range can be expressed as the set of all $\mathbf{n}$ with elements $n_{i}$ satisfying

$$
\min _{d_{j}=0 \text { or } 1} \sum_{j=0}^{D-1} M_{i j} d_{j} \leq n_{i} \leq \max _{d_{j}=0 \text { or } 1} \sum_{j=0}^{D-1} M_{i j} d_{j}
$$

or

$$
\sum_{\substack{j=0 \\ M_{i j}<0}}^{D-1} M_{i j} \leq n_{i} \leq \sum_{\substack{j=0 \\ M_{i j} \geq 0}}^{D-1} M_{i j}
$$

where $M_{i j}$ denotes the $(i, j)$ th element of $\mathbf{M}$. However, for some $\mathbf{M}$, this searching range may be very large even though $J(\mathbf{M})$ is small. As proposed in [32], the Smith form of $\mathbf{M}$ can solve this problem. Let $\mathbf{M}=\mathbf{U} \mathbf{\Lambda V}$ be a Smith form of $\mathbf{M}$. We first compute all integer vectors $\mathbf{b}$ in $\mathscr{N} \mathbf{\Lambda})$. This is straightforward because we simply list all b with integer elements $0 \leq b_{i} \leq \lambda_{i}-1$. We then compute all $((\mathbf{U b}))_{\mathbf{M}}$ to obtain elements in $\mathscr{N}(\mathbf{M})$, i.e.

$$
\mathscr{N}(\mathbf{M})=\left\{((\mathbf{U b}))_{\mathbf{M}} \mid \mathbf{b} \in \mathscr{N}(\mathbf{\Lambda})\right\} .
$$

Since $((\mathbf{U b}))_{\mathbf{M}}$ belongs to $\mathscr{N}(\mathbf{M})$ and $J(\mathbf{M})=J(\mathbf{\Lambda})$, we only need to show that all $((\mathbf{U b}))_{M}$ are distinct to prove (59). This proof is given in Appendix III.

\section{Properties of INTEger MATrices}

The concepts of coprimeness, greatest common divisor ( $\mathrm{gcd}$ ), and least common multiple $(\mathrm{lcm})$ of integers, very often appear in the contexts of 1-D multirate systems [3], [55], [56]. To extend these results to the MD case, we need similar concepts for the case of integer matrices because of the important roles played by the decimation/expansion matrices (which are integer matrices). In system theory, many of these concepts have been derived for polynomial matrices. Some examples are: the greatest common left/right divisors (gcld/gcrd), right/left coprimeness, irreducible right/left matrix fraction descriptions (MFD), the Smith form, and the Smith-McMillan form [57]-[59]. For the integer-matrix case, these concepts, including least common right/left multiples (lcrm/ $\mathrm{lclm})$, have been discussed in [46], [59]-[61]. The purpose of this section is to introduce some other properties of integer matrices, which will be applied in deriving many results in the following sections. We first give the definitions of $1 \mathrm{crm} / \mathrm{lclm}$, which are slightly different from those given in [60], but more proper for our discussions:

\section{Definitions:}

1) $\mathbf{R}$ is a common right multiple of $\mathbf{M}$ and $\mathbf{L}$ (denoted as $\operatorname{crm}(\mathbf{M}, \mathbf{L})$ ) if $\mathbf{R}=\mathbf{M P}=\mathbf{L Q}$ for some integer matrices $\mathbf{P}$ and $\mathbf{Q}$. 


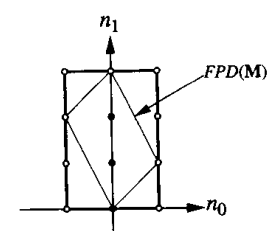

Fig. 25. Circumscribing rectangular of $F P D(\mathbf{M})$.

2) $\mathbf{R}_{0}$ is a least common right multiple of $\mathbf{M}$ and $\mathbf{L}$ (denoted as $\operatorname{lcrm}(\mathbf{M}, \mathbf{L})$ ) if

a) $\mathbf{R}_{0}$ is a nonsingular $\mathrm{crm}(\mathbf{M}, \mathbf{L})$.

b) If $\mathbf{R}$ is another nonsingular $\operatorname{crm}(\mathbf{M}, \mathbf{L})$, then $\mathbf{R}=$ $\mathbf{R}_{0} \mathbf{S}$ for some integer matrix $\mathbf{S}$.

Remark: The common left multiple (clm) and least common left multiple (lclm) are defined similarly.

From the above definitions, it can be verified that an lcrm is a nonsingular crm with the smallest absolute determinant; however, in general it is not unique. In particular, it can be shown that the $\operatorname{lcm}(\mathbf{M}, \mathbf{L})$ is unique up to postmultiplication by a unimodular matrix. We can also prove the following results [31], [36], [39]:

Theorem 2: Let $\mathbf{R}$ be a nonsingular $\operatorname{crm}(\mathbf{M}, \mathbf{L})$, i.e., $\mathbf{R}=\mathbf{M P}=\mathbf{L Q}$. Then, $\mathbf{R}$ is an $\operatorname{lcrm}(\mathbf{M}, \mathbf{L})$ if and only if $\mathbf{P}$ and $\mathbf{Q}$ are right coprime.

Theorem 3: When $\mathbf{M L}=\mathbf{L} \mathbf{M}$, any $\operatorname{lcrm}(\mathbf{M}, \mathbf{L})$ and any $\operatorname{gcrd}(\mathbf{M}, \mathbf{L})$ can be related as $\operatorname{lcrm}(\mathbf{M}, \mathbf{L}) \cdot \mathbf{U} \cdot \operatorname{gcrd}(\mathbf{M}, \mathbf{L})=$ ML for some unimodular U. Also, when $\mathbf{M L}=\mathbf{L M}$, the following four statements are equivalent:

1) $\mathbf{M L}$ is an $\operatorname{lcrm}(\mathbf{M}, \mathbf{L})$.

2) $\mathbf{M}$ and $\mathbf{L}$ are right coprime.

3) $\mathbf{M}$ and $\mathbf{L}$ are left coprime.

4) $\mathbf{M L}$ is an $\operatorname{lclm}(\mathbf{M}, \mathbf{L})$.

Remark: For the 1-D case, this nicely reduces to "lcm $(M, L)=M L$ if and only if $M$ and $L$ are coprime," which is a well-known fact.

\section{Computation of lcrm/lclm Using the Matrix Fraction Description (MFD)}

The right/left irreducible MFD's [58], [59] enable us to compute an $\mathrm{lcrm} / \mathrm{lclm}$ of two matrices (which gives a constructive way of proving the existence of a nonsingular $\mathrm{lcrm} / \mathrm{lclm}$ of two nonsingular matrices). To compute an lcrm of nonsingular $\mathbf{M}$ and $\mathbf{L}$, we let $\mathbf{H}=\mathbf{M}^{-1} \mathbf{L}$ (which is also nonsingular) and compute one irreducible right MFD of $\mathbf{H}$, so we have $\mathbf{M}^{-1} \mathbf{L}=\mathbf{P}_{1} \mathbf{Q}_{1}^{-1}$, where $\mathbf{P}_{1}$ and $\mathbf{Q}_{1}$ are right coprime. Therefore, $\mathbf{M} \mathbf{P}_{1}=\mathbf{L} \mathbf{Q}_{1}$. Denote this as $\mathbf{R}$. Using Theorem 2, we can conclude that $\mathbf{R}$ is an $\operatorname{lcrm}(\mathbf{M}, \mathbf{L})$. Similarly, if we let $\mathbf{H}^{\prime}=\mathbf{L M}^{-1}$ and compute one irreducible left MFD, $\mathbf{H}^{\prime}=\mathbf{Q}_{2}^{-1} \mathbf{P}_{2}$, then $\mathbf{R}^{\prime}=\mathbf{P}_{2} \mathbf{M}=\mathbf{Q}_{2} \mathbf{L}$ is an $\operatorname{lclm}(\mathbf{M}, \mathbf{L})$.

Using the concepts presented in this section, various MD multirate problems can be resolved. In Sections VI-VIII we will outline some of these issues. Interested readers can see [31], [36], and [39] for more details.

\section{COMMUTATIVITY OF DECIMATORS AND EXPANDERS, AND APPLICATION TO RATIONAL DECIMATION SYSTEMS}

For the 1-D case, an $M$-fold decimator and an $L$-fold expander can be interchanged without changing the inputoutput relation if and only if $M$ and $L$ are relatively prime [3]. Conditions for such interchangeability in the 2-D case were first addressed in [27] for upper triangular $\mathbf{M}$ and $\mathbf{L}$. Since then, several other research groups have been trying to improve the results in [27] and to extend them to the MD case. Conditions with different degree of usefulness were found in [31]-[33], [39], [42], [43], and [66]. For a summary of these results, please read [39, sec. VIII]. In this section, we shall present the conditions given in [31] and [39], which are explicit and easy to test. The conditions we shall derive work for any $\mathbf{M}, \mathbf{L}$, and any number of dimensions.

Consider $y_{1}(\mathbf{n})$ and $y_{2}(\mathbf{n})$ in Fig. 26(a) and (b). From the definitions of $\mathbf{M}$-fold decimator and $\mathbf{L}$-fold expander, we have

$$
y_{1}(\mathbf{n})= \begin{cases}x\left(\mathbf{M L}^{-1} \mathbf{n}\right), & \mathbf{n} \in \operatorname{LAT}(\mathbf{L}) \\ 0, & \text { otherwise }\end{cases}
$$

and

$$
y_{2}(\mathbf{n})= \begin{cases}x\left(\mathbf{L}^{-1} \mathbf{M n}\right), & \mathbf{M n} \in \operatorname{LAT}(\mathbf{L}) \\ 0, & \text { otherwise }\end{cases}
$$

For $y_{1}(\mathbf{n})$ to be identical to $y_{2}(\mathbf{n})$, we should in particular have $x\left(\mathbf{M L}^{-1} \mathbf{n}\right)=x\left(\mathbf{L}^{-1} \mathbf{M n}\right)$ for $\mathbf{n} \in \operatorname{LAT}(\mathbf{L})$. Because $x(\mathbf{n})$ is arbitrary, we should have

$$
\mathbf{M L}^{-1} \mathbf{n}=\mathbf{L}^{-1} \mathbf{M n}, \quad \forall \mathbf{n} \in \operatorname{LAT}(\mathbf{L}) .
$$

This implies $\mathbf{M L} \mathbf{L}^{-1}=\mathbf{L}^{-1} \mathbf{M}$, i.e., $\mathbf{L M}=\mathbf{M L}$.

In order for $y_{1}(\mathbf{n})=y_{2}(\mathbf{n})$, we also need

$$
\mathbf{n} \in L A T(\mathbf{L}) \text { if and only if } \mathbf{M n} \in L A T(\mathbf{L}) .
$$

Let $\mathbf{k}=\mathbf{M n}$. It is easily verified that $\mathbf{n} \in L A T(\mathbf{L})$ if and only if $\mathbf{k} \in L A T(\mathrm{ML})$. For the right-hand side, since $\mathbf{k}=$ Mn is also in $L A T(\mathbf{M})$, we can see $M n \in L A T(\mathbf{L})$ if and only if $\mathbf{k} \in L A T(\mathbf{L}) \cap L A T(\mathbf{M})$. It has been shown that $L A T(\mathbf{L}) \cap L A T(\mathbf{M})=L A T(\operatorname{lcrm}(\mathbf{M}, \mathbf{L})) \quad[39$, appendix $]$. Therefore, the condition in (63) is equivalent to

$$
\mathbf{k} \in L A T(\mathbf{M L}) \quad \text { if and only if } \mathbf{k} \in L A T(\operatorname{lcrm}(\mathbf{M}, \mathbf{L}))
$$

which implies that ML is an $\operatorname{lcrm}(\mathbf{M}, \mathbf{L})$.

Combining the above discussion and Theorem 3, we can conclude the following:

Theorem 4: The $\mathbf{L}$-fold expander and the $\mathbf{M}$-fold decimator can be interchanged if and only if 1$) \mathbf{M L}=\mathbf{L M}$ and 2) $\mathbf{M}$ and $\mathbf{L}$ are coprime.

Remark: Note that in general we have to distinguish right coprimeness with left coprimeness for the matrix case. However, Theorem 3 ensures that when $\mathbf{M L}=\mathbf{L M}$, right coprimeness and left coprimeness imply each other. 


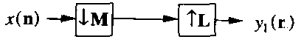

(a)

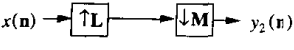

(b)

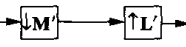

(c)

Fig. 26. Interchange of an MD decimator and an MD expander.

A similar, but more relaxed, commutativity has also been considered [33], [43]. This is shown in Fig. 26(b) and (c). In [43], it was shown that when $\mathbf{M}$ and $\mathbf{L}$ are left coprime, there exist $\mathbf{M}^{\prime}$ and $\mathbf{L}^{\prime}$, which are right coprime, such that the system in Fig. 26(b) is equivalent to the one in Fig. 26(c). Inspired by this, we came up with the following result, which can be called the relaxed commutativity [39]:

Theorem 4.1: Given the matrices $\mathbf{M}$ and $\mathbf{L}$ in Fig. 26(b), we can always find $\mathbf{M}^{\prime}$ and $\mathbf{L}^{\prime}$ in Fig. 26/c) such that these two systems are equivalent by computing an irreducible right MFD of $\mathbf{L}^{-1} \mathbf{M}$, i.e., $\mathbf{L}^{-1} \mathbf{M}=\mathbf{M}^{\prime} \mathbf{L}^{\prime-1}$ and $\mathbf{M}^{\prime}$ and $\mathbf{L}^{\prime}$ are right coprime. Conversely, given right-coprime matrices $\mathbf{M}^{\prime}$ and $\mathbf{L}^{\prime}$ in Fig. 26(c), we can always find $\mathbf{M}$ and $\mathbf{L}$ in Fig. 26(b) such that these two systems are equivalent by computing a left MFD (not necessarily irreducible) of $\mathbf{M}^{\prime} \mathbf{L}^{-1}$, i.e., $\mathbf{M}^{\prime} \mathbf{L}^{-1}=\mathbf{L}^{-1} \mathbf{M}$.

\section{A. Rational Decimation Systems}

Next, we shall apply the commutativity of decimators and expanders to the implementation of MD rational decimation systems. An MD decimation system with rational decimation ratio (in this case, a matrix) $\mathbf{H}=\mathbf{L}^{-1} \mathbf{M}$ is shown in Fig. 27. As in the 1-D case, the MD filter $H(\mathbf{z})$ in Fig. 27 is used to suppress image components generated by the $\mathbf{L}$-fold expander and eliminate aliasing due to the $\mathbf{M}$-fold decimator. We usually assume that $\mathbf{M}$ and $\mathbf{L}$ are left coprime. In fact, if they were not ieft coprime, any common left divisor of them could be canceled without changing the overall decimation ratio $\mathbf{H}$. Rational decimation systems find applications in the conversion of images or video data between different sampling standards. As an example, if

$$
\mathbf{L}=\left[\begin{array}{ll}
2 & 0 \\
0 & 1
\end{array}\right] \quad \mathbf{M}=\left[\begin{array}{rr}
1 & 1 \\
1 & -1
\end{array}\right]
$$

we can convert rectangularly sampled inages to hexagonally sampled ones, as shown in [7, fig. 6]. Rational decimation systems can also be used to reduce the data rate of bandlimited signals [67].

Remark: We often want a rational decimation system to preserve the values of existing samples in the input. In other words, in Fig. 27 we want $y(\mathbf{n})=x(\mathbf{m})$ whenever $\mathbf{M n}=\mathbf{L m}$. It can be shown that this is true if and only if $H(\mathbf{z})$ is an $\mathbf{L}$ th-band Nyquist filter, whether $\mathbf{M}$ and $\mathbf{L}$ are left coprime or not. It turns out that the $\mathbf{M}$-fold decimator is not significant here.

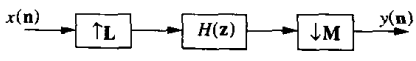

Fig. 27. MD rational decimation system.

Recall that a 1-D sampling-rate alteration system with decimation ratio $M / L$ can be implemented efficiently by using RPI when $M$ and $L$ are coprime [54]. We now extend this idea to the MD case. We first use the MD polyphase decomposition to implement the system of Fig. 27 as in Fig. 28 (figures only show the case where $J(\mathbf{L})=2$ and $J(\mathbf{M})=3$ ). Next, we shall derive the so-called MD RPI technique to improve the efficiency further. Fig. 29 shows this by successively redrawing the circuit of Fig. 28.

Starting from Fig. 28, suppose it is possible to replace every $\mathbf{k}_{i}$ in $\mathscr{N}(\mathbf{M})$ with $\mathbf{M k}_{i 1}+\mathbf{L} \mathbf{k}_{i 2}$, where $\mathbf{k}_{i 1}$ and $\mathbf{k}_{i 2}$ are some integer vectors. This is indeed true if $\mathbf{M}$ and $\mathbf{L}$ are left coprime [39]. Hence we get Fig. 29(a). With the help of Noble identities, Fig. 29(a) can be redrawn as Fig. 29 (b). Next, by using Theorem 4.1, we can interchange the expanders and decimators and replace them with $\mathbf{M}^{\prime}$ and $\mathbf{L}^{\prime}$, where $\mathbf{M}^{\prime}$ and $\mathbf{L}^{\prime}$ form an irreducible right $\mathrm{MFD}$ of $\mathbf{L}^{-1}$ M. Hence we obtain Fig. 29(c). We can then perform Type 2 polyphase decomposition on $E_{i}(\mathbf{z})$ 's with respect to $\mathbf{L}^{\prime}$, and get Fig. 29(d). It can be verified that $E_{i, j}$ (z)'s are the $\mathbf{M L}^{\prime}$-fold (or $\mathbf{L} \mathbf{M}^{\prime}$-fold) polyphase components of $H(\mathbf{z})$, so the total number of arithmetic operations in $E_{i, j}(\mathbf{z})$ 's is the same as the number in $H(\mathbf{z})$. However, each arithmetic operation in Fig. 29(d) is now performed at its lowest rate, which is $1 / J\left(\mathbf{M}^{\prime}\right) J(\mathbf{L})$ times the rate of $H(\mathbf{z})$ in Fig. 27.

\section{B. An Alternative}

We now present an alternative to the MD polyphase technique mentioned above. Suppose we need to decimate an MD signal by a rational nonsingular matrix $\mathbf{H}$. Using the Smith-McMillan form [58], [59], we obtain $\mathbf{H}=\mathbf{U} \boldsymbol{\Lambda} \mathbf{V}$ where $\mathbf{U}$ and $\mathbf{V}$ are unimodular integer matrices and $\boldsymbol{\Lambda}=\operatorname{diag}\left[\lambda_{0}, \cdots, \lambda_{D-1}\right]$. The $\lambda_{i}$ 's are nonzero rational numbers, i.e., ratios of integers. We represent all $\lambda_{i}$ 's in their irreducible forms, i.e., $\lambda_{i}=\alpha_{i} / \beta_{i}$, where $\alpha_{i}$ and $\beta_{i}$ are coprime integers. Let $\Lambda_{\alpha}=\operatorname{diag}\left[\alpha_{0}, \cdots, \alpha_{D-1}\right]$ and $\Lambda_{\beta}=\operatorname{diag}\left[\beta_{0}, \cdots, \beta_{D-1}\right]$ so that

$$
\mathbf{H}=\mathbf{U} \boldsymbol{\Lambda}_{\beta}^{-1} \boldsymbol{\Lambda}_{\alpha} \mathbf{V} \text {. }
$$

Instead of using the system in Fig. 27, the rational decimation can be accomplished using the system in Fig. 30 . Now, since $\boldsymbol{\Lambda}_{\alpha}$ and $\boldsymbol{\Lambda}_{\beta}$ are both diagonal, the filter $H(\mathbf{z})$ can be designed separately in each dimension, i.e., $H(\mathbf{z})$ $=H_{0}\left(z_{0}\right) \cdots H_{D-1}\left(z_{D-1}\right)$. (Note that because $H(\mathbf{z})$ is restricted to be separable, some flexibility is lost by using this approach.) In the $i$ th dimension, $H_{i}\left(z_{i}\right)$ is chosen to suppress images due to the $\beta_{i}$-fold expansion and eliminate aliasing owing to the $\alpha_{i}$-fold decimation. Hence, the overall system will be free from aliasing and no images exist. Finally, we can use the 1-D rational polyphase technique described in [54] to implement each $H_{i}\left(z_{i}\right)$ efficiently because $\alpha_{i}$ and $\beta_{i}$ are coprime. 


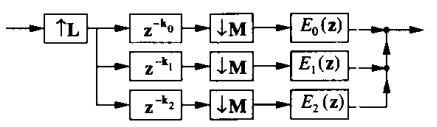

Fig. 28. Polyphase implementation of an MD rational decimation system.

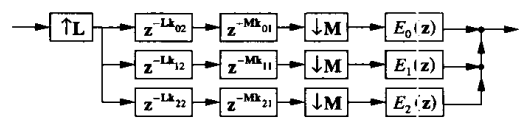

(a)

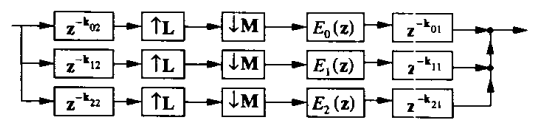

(b)

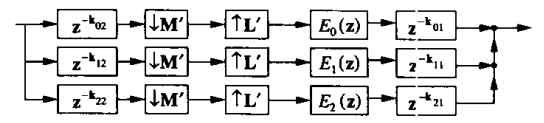

(c)

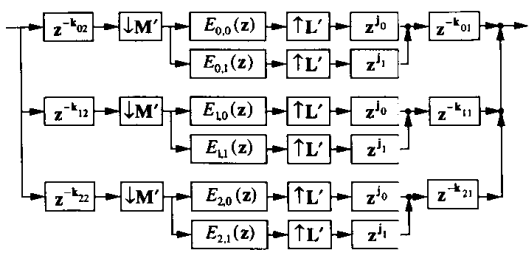

(d)

Fig. 29. Successive redrawing of polyphase implementations of an MD rational decimation system.

$$
\stackrel{x(\mathbf{n})}{\longrightarrow} \downarrow \mathbf{U}-\uparrow \mathbf{\Lambda}_{\beta}-H(\mathbf{z})-\downarrow \mathbf{\Lambda}_{a}-\downarrow \overline{\mathbf{V}}{ }^{y(\mathbf{n})}
$$

Fig. 30. MD rational decimation system using Smith-McMillan form.

\section{MD DELAY-ChaIN SySTEMS}

A 1-D delay-chain system as shown in Fig. 13 is a PR system, i.e., $\hat{x}(n)=x(n)$, if and only if $M$ and $L$ are coprime. This was proved in [55], and applications of this PR system in the design of filter-bank systems can also be found therein. We shall now extend this concept to the MD case. One potential application of MD delay-chain systems is to design MD filter banks with the analysis and synthesis filters having a certain symmetry.

An MD delay-chain system is shown in Fig. 31. (The case of $\mathbf{L}=\mathbf{I}$ is most commonly used and is a PR filter bank.) We can see that this is a very special case of MD maximally decimated filter banks with $J(\mathbf{M})$ channels, where the analysis and synthesis filters are only shift operators (sometimes called "delays") defined as

$$
H_{i}(\mathbf{z})=\mathbf{z}^{-\mathbf{L} \mathbf{k}_{i}} \quad \text { and } \quad F_{i}(\mathbf{z})=z^{\mathbf{L k}_{i}}, \quad \text { for } \mathbf{k}_{i} \in \mathscr{N}(\mathbf{M}) .
$$

Using the definitions of decimation and expansion, we can write the signals $v_{i}(\mathbf{n})$ in Fig. 31 as follows:

$$
v_{i}(\mathbf{n})= \begin{cases}x(\mathbf{n}), & \mathbf{n}+\mathbf{L k}_{i} \in L A T(\mathbf{M}) \\ 0, & \text { otherwise }\end{cases}
$$

Since $\hat{x}(\mathbf{n})=\sum_{\mathbf{k}_{i} \in \mathscr{N}(\mathbf{M})} v_{i}(\mathbf{n})$, so $\hat{x}(\mathbf{n})=x(\mathbf{n})$ if and only if

$$
\bigcup_{\mathbf{k}_{i} \in \mathcal{N}(\mathbf{M})}\left\{\mathbf{n} \mid \mathbf{n}+\mathbf{L} \mathbf{k}_{i} \in L A T(\mathbf{M})\right\}=\mathscr{N} .
$$

It can be verified that the above condition is true if and only if

$$
\underbrace{\left\{\left(\left(\mathbf{L} \mathbf{k}_{i}\right)\right)_{\mathbf{M}} \mid \mathbf{k}_{i} \in \mathscr{N}(\mathbf{M})\right\}}_{\mathscr{S}}=\mathscr{N}(\mathbf{M}) .
$$

Because of the modulo notation, all the elements in $\mathscr{S}$ are also in $\mathscr{N}(\mathbf{M})$. Therefore, (69) is true if and only if all $\left(\left(\mathbf{L k}_{i}\right)\right)_{\mathbf{M}}$ 's (for $\left.\mathbf{k}_{i} \in \mathscr{N}(\mathbf{M})\right)$ are distinct. The following theorem [39] gives the sufficient condition for this:

Theorem 5: If $\mathbf{L M}$ is an $\operatorname{lcrm}(\mathbf{L}, \mathbf{M})$, then $\mathscr{S}=\mathcal{N}(\mathbf{M})$. Hence, the MD delay chain is PR.

This is not a necessary condition, though. The problem of finding the necessary and sufficient conditions for PR delay-chain systems is still open. However, if we assume $\mathbf{M L}=\mathbf{L M}$, we can show the following [39]:

Theorem 6: If $\mathbf{M L}=\mathbf{L M}$, then the condition that $\mathbf{L M}$ is an $\operatorname{lcrm}(\mathbf{L}, \mathbf{M})$ becomes necessary and sufficient for the delay-chain system in Fig. 31 to achieve PR.

\section{Periodicity Matrices of Decimated SIGNALS}

It is well-known that in the $1-\mathrm{D}$ case, a signal with period $P$ is also periodic with period $P S$ where $S$ is any nonzero integer. In MD, a similar fact is true. Let $x(\mathbf{n})$ have periodicity matrix $\mathbf{P}$. If $\mathbf{Q}=\mathbf{P S}$ for some integer matrix $\mathbf{S}$, then $\mathbf{Q}$ is also a periodicity matrix of $x(\mathbf{n})[49, \mathrm{p}$. 12]. Since the periodicity matrix of an MD signal is not unique, we are usually interested in those with the smallest absolute determinant. (We exclude the case of singular periodicity matrices.)

It is well-known that when a $1-\mathrm{D}$ periodic signal $x(n)$ with period $L$ is decimated by a factor of $M$ to obtain $y(n)=x(M n)$, the period of $y(n)$ is $L / \operatorname{gcd}(M, L)=$ $\operatorname{lcm}(M, L) / M$. We now consider the following question: When an MD signal $x(\mathbf{n})$ with periodicity matrix $\mathbf{L}$ is decimated by $\mathbf{M}$, is the output $y(\mathbf{n})=x(\mathbf{M n})$ periodic? If yes, what is the periodicity matrix? In other words, given $x(\mathbf{n}+\mathbf{L k})=x(\mathbf{n}), \forall \mathbf{k} \in \mathscr{N}$, we want to find $\mathbf{P}$ such that $y(\mathbf{n}+\mathbf{P k})=y(\mathbf{n}), \quad \forall \mathbf{k} \in \mathscr{N}$. Since $y(\mathbf{n}+\mathbf{P k})=x(\mathbf{M n}+$ $\mathbf{M P k})$, we can see that $y(\mathbf{n}+\mathbf{P k})=y(\mathbf{n})$ if $\mathbf{M P k}=\mathbf{L q}$ for some $\mathbf{q} \in \mathscr{N}$. Therefore, $\mathbf{P}$ is a periodicity matrix of $y(\mathbf{n})$ if

$$
\forall \mathbf{k} \in \mathscr{N}, \quad \exists \mathbf{q} \in \mathscr{N}, \quad \text { such that } \quad \mathbf{M P k}=\mathbf{L q} .
$$

It can be verified that this condition is equivalent to

$$
\mathbf{M P}=\mathbf{L Q} \text { for some integer matrix } \mathbf{Q}
$$

i.e., $\mathbf{M P}=\mathbf{L Q}$ is a $\operatorname{crm}(\mathbf{M}, \mathbf{L})$. Thus, $\mathbf{P}$ is a periodicity matrix of $y(\mathbf{n})$ if $\mathbf{P}=\mathbf{M}^{-1} \operatorname{crm}(\mathbf{M}, \mathbf{L})$. Clearly, $\mathbf{P}$ is not unique. Moreover, using the notation of $1 \mathrm{crm}$, we can conclude that the nonsingular $\mathbf{P}$ that satisfies (71) with the 


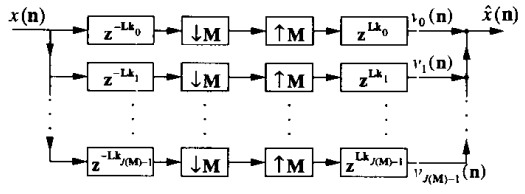

Fig. 31. MD delay-chain systern.

smallest absolute determinant is $\mathbf{P}=: \mathbf{M}^{-1} \operatorname{lcrm}(\mathbf{M}, \mathbf{L})$. Note that this is a sufficient condition for $\mathbf{P}$ to be a periodicity matrix of $y(\mathbf{n})$. If further knowledge about $x(\mathbf{n})$ is available, a periodicity matrix with even smaller absolute determinant can be found.

\section{A. Case of Cyclo-Wide-Sense-Stationary Signals}

The result for the statistical case is similar. We shall first define the cyclo-wide-sense-stationary (CWSS) property for MD statistical processes (random signals) as follows:

Definition: Let $R_{x x}(\mathbf{n}, \mathbf{m})=E\left[x(\mathbf{n}) x^{*}(\mathbf{n}-\mathbf{m})\right]$ denote the autocorrelation function of an MD process, where $E[\cdot]$ is the statistical mean. The process $x(\mathbf{n})$ is said to be CWSS with periodicity matrix $\mathbf{L}$ (denoted as $(\mathrm{CWSS})_{\mathrm{L}}$ ) if

1) $E[x(\mathbf{n})]=E[x(\mathbf{n}+\mathbf{L k})], \forall \mathbf{k} \in \mathscr{N}$, i.e., $E[x(\mathbf{n})]$ is periodic with periodicity matrix $\mathbf{L}$.

2) $R_{x x}(\mathbf{n}, \mathbf{m})=R_{x x}(\mathbf{n}+\mathbf{L k}, \mathbf{m}), \quad \forall \mathbf{k}, \mathbf{m} \in \mathscr{N}$, i.e., $R_{x x}(\mathbf{n}, \mathbf{m})$ is periodic in $\mathbf{n}$ with periodicity matrix $\mathbf{L}$.

Now, given that $x(\mathbf{n})$ is $(\mathrm{CWSS})_{\mathbf{L}}$, let $y(\mathbf{n})=x(\mathbf{M n})$. What can we say about the cyclo-stationarity of $y(\mathbf{n})$ ? We know $E[y(\mathbf{n})]=E[x(\mathbf{M n})]$ and $R_{y y}(\mathbf{n}, \mathbf{m})=E\left[y(\mathbf{n}) y^{*}\right.$ $(\mathbf{n}-\mathbf{m})]=E\left[x(\mathbf{M n}) x^{*}(\mathbf{M n}-\mathbf{M m})\right]=R_{x x}(\mathbf{M n}, \mathbf{M m})$. That is, $E[y(\mathbf{n})]$ can be obtained by M-fold decimating $E[x(\mathbf{n})]$, and $R_{y y}(\mathbf{n}, \mathbf{m})$ can be obtained by M-fold decimating $R_{x x}(\mathbf{n}, \mathbf{m})$ with respect to both $\mathbf{n}$ and $\mathbf{m}$. Using the result we obtained for the deterministic case, we can conclude that $E[y(\mathbf{n})]$ has periodicity matrix $\mathbf{P}=$ $\mathbf{M}^{-1} \operatorname{lcrm}(\mathbf{M}, \mathbf{L})$, and $R_{y y}(\mathbf{n}, \mathbf{m})$ also has the same periodicity matrix with respect to $\mathbf{n}$. (Note that the decimation with respect to the second argument $\mathbf{m}$ is not significant here.) Therefore, $y(\mathbf{n})$ is $(\mathrm{CWSS})_{\mathbf{P}}$ with $\mathbf{P}=\mathbf{M}^{-1}$ $\operatorname{lcrm}(\mathbf{M}, \mathbf{L})$

The above results are summarized in Fig. 32. Note that for the $1-\mathrm{D}$ case, these nicely reduce to $P^{\prime}=\operatorname{lcm}(M, L) / M$ [56].

\section{B. Fundamental Periodicity Matrices}

As mentioned earlier, the periodicity matrix of an MD signal is not unique and we are usually interested in those with the smallest absolute determinant. For this reason, one defines the fundamental periodicity matrix as follows:

Definition: $\mathbf{P}_{0}$ is a fundamental periodicity matrix of $x(\mathbf{n})$ if

1) $\mathbf{P}_{0}$ is a periodicity matrix of $x(\mathbf{n})$

2) Any other periodicity matrix of $x(\mathbf{n})$, say $\mathbf{P}$, can be written as $\mathbf{P}=\mathbf{P}_{0} \mathbf{S}$ for some integer matrix $\mathbf{S}$. That is, $\mathbf{P}_{0}$ is a left divisor of all the periodicity matrices of $x(\mathbf{n})$.

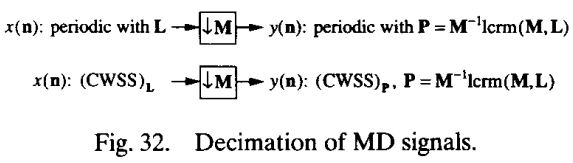

From this definition, a fundamental periodicity matrix of an MD signal is indeed a periodicity matrix with the smallest absolute determinant (with singular periodicity matrices excluded).

\section{Alias-Free Decimation}

As in the 1-D case, the MD decimation creates aliasing if the input signal is not "properly bandlimited." Therefore, a decimation filter $H_{F}(\boldsymbol{\omega})$ is usually used to prefilter the input signals, as in Fig. 6(a). For the 1-D case, it has been shown that the aliasing is completely avoided if and only if the support of the decimation filter $H_{F}(\omega)$ does not overlap under the modulo- $2 \pi / M$ operation [56]. As in the 1-D case, we can define the MD modulo-V operation as "folding the support (in the range $[-\pi, \pi)^{D}$ ) of a filter into the region $F P D(V)$ by translating each component of the support by $\mathbf{V k}$ for some integer vector $\mathbf{k}$."

Using the frequency-domain relation of decimation (5), we can obtain the following condition of alias-free decimation:

Fact 1: The decimation system in Fig. 6(a) is alias free if and only if the support of the decimation filter $H_{F}(\omega)$ does not overlap under the modulo- $2 \pi \mathbf{M}^{-T}$ operation.

Remark: A weaker condition has been addressed in [22]. The condition given above is more explicit and gives a more systematic method to test the alias-free property of a given decimation filter.

For example, when

$$
\mathbf{M}=\left[\begin{array}{rr}
2 & -2 \\
2 & 4
\end{array}\right]
$$

Fig. 33 shows some admissible supports of the decimation filter. The arrows show the modulo- $2 \pi \mathbf{M}^{-T}$ operation. Note that in each case, the passband support fits into the region $F P D\left(2 \pi \mathbf{M}^{-T}\right)$ with no overlap. Clearly, $F P D\left(2 \pi \mathbf{M}^{-T}\right)$ itself is an alias-free support. The following relation between $S P D(\cdot)$ and $F P D(\cdot)$ can be verified

$$
S P D(\mathbf{V})=F P D(2 \mathbf{V})-\mathbf{V}\left[\begin{array}{lll}
1 & \cdots & 1
\end{array}\right]^{T}
$$

The expression on the right-hand side means that all elements in $F P D(2 \mathbf{V})$ are subtracted by $\mathbf{V}\left[\begin{array}{lll}1 & \cdots & 1\end{array}\right]^{T}$. In other words, $S P D(\mathbf{V})$ can be obtained by fist scaling $F P D(\mathrm{~V})$ up by 2 in each dimension and then shifting it by $-V\left[\begin{array}{lll}1 & \cdots & 1\end{array}\right]^{T}$. It is easily verified that any shifted version of an alias-free support is also alias-free. Therefore, we conclude that $\operatorname{SPD}\left(\pi \mathbf{M}^{-T}\right)$ is also an alias-free support. Fig. 33(b) shows exactly this region for the above chosen M. In practical applications, although there are an infinite number of alias-free supports for the decimation filter, $\operatorname{SPD}\left(\pi \mathbf{M}^{-T}\right)$ is the typical choice. 


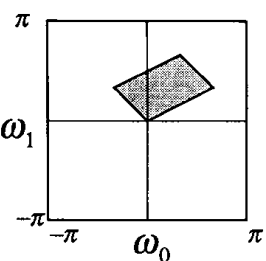

(a)

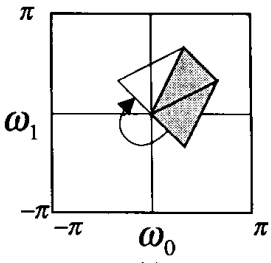

(c)

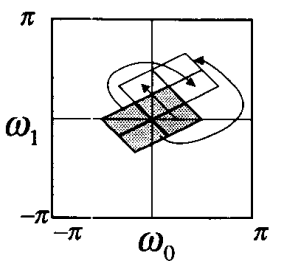

(b)

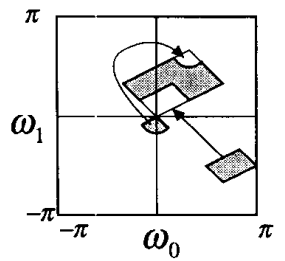

(d)
Fig. 33. Alias-free passband supports for the decimation matrix in (72).

Remark: Since expansion introduces image components, an interpolation filter is required to suppress these images, as in Fig. 6(b). From (9) we see that in the L-fold expansion, the input spectrum in the range $[-\pi, \pi)^{D}$ (i.e., $\operatorname{SPD}(\pi \mathrm{I})$ ) is mapped to $\operatorname{SPD}\left(\pi \mathbf{L}^{-T}\right)$. Any other components outside $S P D\left(\pi \mathbf{L}^{-T}\right)$ are images. Therefore, a typical choice for the interpolation filter is $\operatorname{SPD}\left(\pi \mathbf{L}^{-T}\right)$.

\section{Multistage Design of Multirate Systems}

As mentioned earlier, interpolated finite-impulseresponse (IFIR) filters [62] are closely related to multistage decimation systems for the $1-D$ case $[1$, sec. 5.1$]$. The same multistage idea can be extended to the MD case. It has been shown that by factorizing a matrix $\mathbf{M}$ into triangular factors $\mathbf{T}_{1} \mathbf{T}_{2} \cdots \mathbf{T}_{K}$, we can get all the multistage implementations of an $\mathbf{M}$-fold decimator [68]. Fig. 34(a) shows an MD multistage decimation system, which results in the overall decimation by $\mathbf{M}=\mathbf{M}_{1} \mathbf{M}_{2}$. The associated filtering is accomplished in two stages. The filters $H_{1}(\mathbf{z})$ and $H_{2}(\mathbf{z})$ are required to ensure that $x_{1}(\mathbf{n})$ and $x_{2}(\mathbf{n})$ are appropriately bandlimited to avoid aliasing. We can rearrange the structure using Noble identities as shown in Fig. 34(b). The overall decimation filter is therefore $H_{1}(\mathbf{z}) H_{2}\left(\mathbf{z}^{\mathbf{M}_{1}}\right)$, or $H_{F, 1}(\boldsymbol{\omega}) H_{F, 2}\left(\mathbf{M}_{1}^{T} \boldsymbol{\omega}\right)$.

As a specific example, let

$$
\mathbf{M}=\left[\begin{array}{rr}
2 & -4 \\
2 & 2
\end{array}\right]=\underbrace{\left[\begin{array}{rr}
1 & -1 \\
1 & 2
\end{array}\right]}_{\mathbf{M}_{1}} \underbrace{\left[\begin{array}{rr}
2 & -1 \\
0 & 2
\end{array}\right]}_{\mathbf{M}_{2}}
$$

If we make the typical choice $S P D\left(\pi \mathbf{M}_{1}^{-T}\right)$ and $S P D$ $\left(\pi \mathbf{M}_{2}^{-T}\right)$ as sketched in Fig. 35(a) for the passband supports of $H_{F, 1}(\omega)$ and $H_{F, 2}(\omega)$, it can be verified that the overall filter $H_{F}(\boldsymbol{\omega})=H_{F, 1}(\boldsymbol{\omega}) H_{F, 2}\left(\mathbf{M}_{1}^{T} \boldsymbol{\omega}\right)$ has the support as in Fig. 35(b). This is exactly $\operatorname{SPD}\left(\pi \mathbf{M}^{-T}\right)$, so the decimation by $\mathbf{M}$ does not cause aliasing.

\section{Subtle Things About Multistage Decimation}

We now consider a different example of decomposition of $\mathbf{M}$, bringing up a situation that does not happen in the

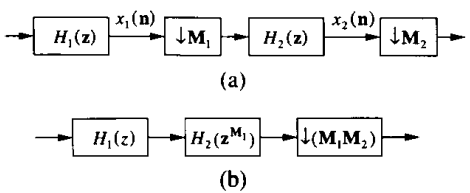

Fig. 34. MD multistage decimation system

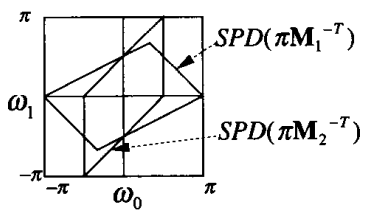

(a)

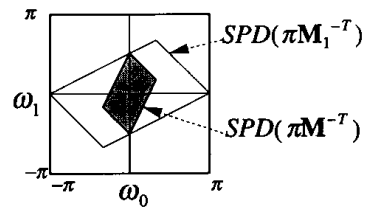

(b)
Fig. 35. Passband supports of an MD multistage decimation system: first example.

1-D case. Let the decimation matrix be

$$
\mathbf{M}=\left[\begin{array}{rr}
1 & -4 \\
1 & 2
\end{array}\right]=\underbrace{\left[\begin{array}{rr}
1 & -1 \\
1 & 2
\end{array}\right]}_{\mathbf{M}_{1}} \underbrace{\left[\begin{array}{rr}
1 & -2 \\
0 & 2
\end{array}\right]}_{\mathbf{M}_{2}}
$$

Fig. 36(a) shows the region $\operatorname{SPD}\left(\pi \mathbf{M}^{-T}\right)$, which is typically taken to be the support of the decimation filter. Suppose we wish to achieve this by multistage design as in Fig. 34 and take the support of $H_{F, 1}(\boldsymbol{\omega})$ as usual, i.e., $\operatorname{SPD}\left(\pi \mathbf{M}_{1}^{-T}\right)$. We see that $\operatorname{SPD}\left(\pi \mathbf{M}^{-T}\right)$ does not even fit inside $S P D\left(\pi \mathbf{M}_{1}^{-T}\right)$. Therefore, the resulting overall filter $H_{F, 1}(\boldsymbol{\omega}) H_{F, 2}\left(\mathbf{M}_{1}^{T} \boldsymbol{\omega}\right)$ (the shaded area in Fig. 36(b)) leaves out part of desired support $\operatorname{SPD}\left(\pi \mathbf{M}^{-T}\right)$ and lets in some band outside $\operatorname{SPD}\left(\pi \mathbf{M}^{-T}\right)$. (However, there is still no aliasing even though the support of the overall filter $H_{F}(\boldsymbol{\omega})$ is not $\operatorname{SPD}\left(\pi \mathbf{M}^{-T}\right)$.) Recall that this situation never happens in the 1-D case because of the fact $M>M_{1}$.

From the above examples, we can see that the problem of the inconsistent overall filter passband arises whenever $\operatorname{SPD}\left(\pi \mathbf{M}^{-T}\right)$ is not contained in $\operatorname{SPD}\left(\pi \mathbf{M}_{1}^{-T}\right)$. The following fact can be verified easily:

Fact 2: The inconsistent-support problem does not exist if and only if $S P D\left(\mathbf{M}_{2}^{-T}\right)$ is contained within the range $[-1,1)^{D}$.

Hence, to avoid this problem, we have to either choose the decomposition $\mathbf{M}=\mathbf{M}_{1} \mathbf{M}_{2}$ properly, or we have to choose some other support instead of $\operatorname{SPD}\left(\pi \mathbf{M}_{1}^{-T}\right)$ for $H_{F, 1}(\boldsymbol{\omega})$. For example, we can choose $\mathbf{M}_{2}$ to be a diagonal matrix (with each diagonal element being a common factor of the corresponding column in $\mathbf{M}$ ), then such $\mathbf{M}_{2}$ is guaranteed to satisfy the condition of Fact 2.

\section{CONCLUding Remarks}

In this paper, we have reviewed the fundamentals of MD multirate signal processing and presented some recent as well as new developments in MD multirate systems. We presented a method to derive all parallelepiped-shaped filters in MD multirate applications from an appropriate 1-D filter. We showed that the generalized- 


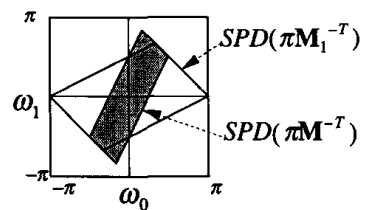

(a)

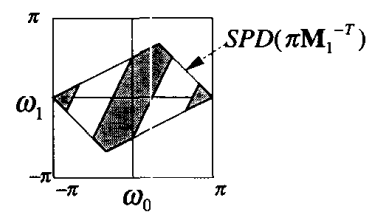

(b)
Fig. 36. Passband supports of an MD multistage: decimation system: second example.

pseudocirculant property is necessary and sufficient for an MD maximally decimated filter-bank system to be free from aliasing. We showed how the Smith form, SmithMcMillan form, and the least common multiples of integer matrices can resolve many nonseparable MD multirate problems. We also mentioned the condition for aliasfree decimation and the multistage design of decimation systems.

There are still many open problems of considerable interest in MD multirate signal processing that remain to be resolved. For example, the problem of designing MD perfect-reconstruction filter banks has not been completely solved. The necessary and sufficient condition for MD delay-chain systems to achieve perfect reconstruction and the applications require further research. A systematic approach to find all possible factorizations of an integer matrix as $\mathbf{M}=\mathbf{M}_{1} \mathbf{M}_{2} \cdots \mathbf{M}_{K}$ that are proper for the multistage decimation, i.e., without inconsistent support problems (Section X), is yet to be found.

\section{APPENDIX I}

The notations and some basics for ML) multirate signal processing are summarized here for quick reference.

\section{Notations}

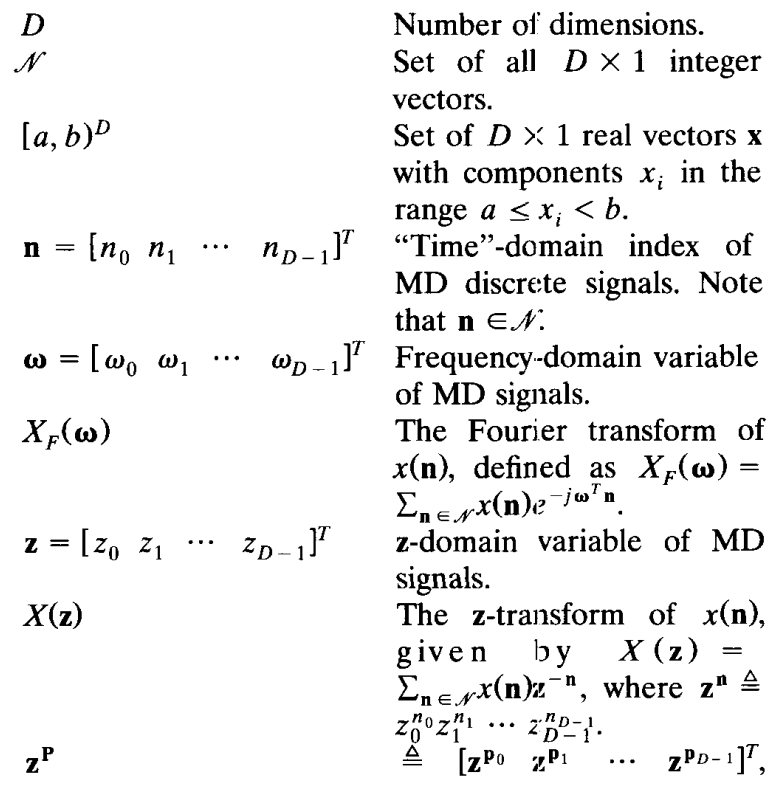
$1-\mathrm{D}$
2-D
MD
DFT
LTI
MFD
PR
RPI
One-dimensional.
Two-dimensional.
Multidimensional.
Discrete Fourier transform.
Linear time invariant.
Matrix fraction description. Perfect reconstruction
Rational polyphase imple- mentation.

where $\mathbf{p}_{i}$ is the $i$ th column of $\mathbf{P}$.

For nonsingular real matrix $\mathbf{V}$ :

1) $L A T(\mathbf{V})$ (lattice generated by $\mathbf{V})$ : set of all vectors of the form $\mathbf{V n}, \mathbf{n} \in \mathscr{N}$.

2) $F P D(\mathbf{V})$ ( fundamental parallelepiped generated by V): set of all real vectors of the form $\mathbf{V x}, \mathbf{x} \in[0,1)^{D}$.

3) $S P D(\mathbf{V})$ (symmetric parallelepiped generated by $\mathbf{V}$ ): set of all real vectors of the form $\mathbf{V x}, \mathbf{x} \in[-1,1)^{D}$. It can be verified that $S P D(\mathbf{V})=F P D(2 \mathbf{V})-$ $\mathbf{V}\left[\begin{array}{lll}1 & \cdots & 1\end{array}\right]^{T}$.

For nonsingular integer matrix $\mathbf{M}$ :

1) $\mathscr{N}(\mathbf{M})$ : set of all integer vectors of the form $\mathbf{M x}$, $\mathbf{x} \in[0,1)^{D}$.

2) $J(\mathbf{M}) \triangleq|\operatorname{det} \mathbf{M}|$ : absolute determinant of $\mathbf{M}$. This is also equal to the number of elements in $\mathscr{N}(\mathbf{M})$.

3) $\hat{\mathbf{M}} \triangleq J(\mathbf{M}) \cdot \mathbf{M}^{-1}= \pm$ [adjugate (adjoint) of $\mathbf{M}$ ], which is also an integer matrix.

\section{Basic Concepts}

1) Periodicity matrix: An MD function $f(\mathbf{x})$ is said to be periodic with periodicity matrix $\mathbf{P}$ if $f(\mathbf{x}+\mathbf{P k})$ $=f(\mathbf{x}), \forall \mathbf{k} \in \mathscr{N}$.

2) The support of a filter $H_{F}(\boldsymbol{\omega})$ is the region of $\boldsymbol{\omega}$ where $H_{F}(\boldsymbol{\omega})$ is nonzero.

3) Zero-phase filters: A filter having purely real frequency response is called a zero-phase filter.

4) Nyquist filters: A Nyquist filter has impulse response $h(\mathbf{n})$ satisfying $h(\mathbf{M n})=0$, for $\mathbf{n} \neq \mathbf{0}$. This is also called a Mth-band filter.

5) Polyphase decomposition: The polyphase components of $x(\mathbf{n})$ with respect to a given $\mathbf{M}$ are defined as

$$
\text { or } \quad \begin{aligned}
& e_{i}(\mathbf{n})=x\left(\mathbf{M n}+\mathbf{k}_{i}\right) \quad(\text { Type } 1) \\
& r_{i}(\mathbf{n})=x\left(\mathbf{M n}-\mathbf{k}_{i}\right) \quad(\text { Type 2) }
\end{aligned}
$$

where $\mathbf{k}_{\mathrm{i}} \in \mathscr{N}(\mathbf{M})$. So $\mathbf{k}_{i}$ can take on $J(\mathbf{M})$ different values, which are ordered as $\mathbf{k}_{0}, \mathbf{k}_{1}, \cdots, \mathbf{k}_{J(\mathbf{M})-1}$. Also, in the $\mathbf{z}$-domain, the polyphase decomposition of $X(\mathbf{z})$ can be expressed as

$$
X(\mathbf{z})=\sum_{\mathbf{k}_{i} \in \mathcal{N}(\mathbf{M})} \mathbf{z}^{-\mathbf{k}_{i}} E_{i}\left(\mathbf{z}^{\mathbf{M}}\right) \quad \text { (Type 1) }
$$

or

$$
X(\mathbf{z})=\sum_{\mathbf{k}_{i} \in \mathcal{N}(\mathbf{M})} \mathbf{z}^{\mathbf{k}_{i}} R_{i}\left(\mathbf{z}^{\mathbf{M}}\right) . \quad \text { (Type 2) }
$$


In the frequency domain, these become

$$
X_{F}(\boldsymbol{\omega})=\sum_{\mathbf{k}_{i} \in \mathcal{N}(\mathbf{M})} e^{-j \omega^{T} \mathbf{k}_{i}} E_{F, i}\left(\mathbf{M}^{T} \boldsymbol{\omega}\right) \quad(\text { Type } 1)
$$

or

$$
X_{F}(\boldsymbol{\omega})=\sum_{\mathbf{k}_{i} \in \mathcal{N}(\mathbf{M})} e^{j \omega^{T} \mathbf{k}_{i}} R_{F, i}\left(\mathbf{M}^{T} \boldsymbol{\omega}\right) . \quad(\text { Type 2) }
$$

6) Noble identities: These are rules that permit us to move decimators and expanders across transfer functions. Figure 20 shows these rules.

7) A linear system is $(L P T V)_{M}$ (linear periodic-timevarying with periodicity matrix $\mathbf{M}$ ) if, given that $y(\mathbf{n})$ is the output for a certain input $x(\mathbf{n}), y(\mathbf{n}+$ Mk) is the output for $x(\mathbf{n}+\mathbf{M k})$ for every $\mathbf{k} \in \mathscr{N}$.

8) Division theorem for integer vectors: For a given nonsingular integer matrix $\mathbf{M}$, every integer vector $\mathbf{n}$ can be uniquely expressed as $\mathbf{n}=\mathbf{M} \mathbf{n}_{0}+\mathbf{k}$ for some $\mathbf{k} \in \mathscr{M}(\mathbf{M})$ and $\mathbf{n}_{0} \in \mathscr{N}$. We denote the "remainder" $\mathbf{k}$ as $\mathbf{k}=\mathbf{n} \bmod \mathbf{M}$, or $\mathbf{k}=((\mathbf{n}))_{M}$.

9) An integer matrix $\mathbf{U}$ is called unimodular if [ $\operatorname{det} \mathbf{U}]$ $= \pm 1$, i.e., $J(\mathbf{U})=1$. For a unimodular matrix $\mathbf{U}$, $\mathbf{U}^{-1}=$ [adjugate of $\left.\mathbf{U}\right] /[\operatorname{det} \mathbf{U}]= \pm$ [adjugate of $\mathrm{U}]$ is also a unimodular integer matrix.

10) An MD random signal $x(\mathbf{n})$ is said to be cyclowide-sense-stationary with periodicity matrix $\mathbf{L}$ (denoted as $(\mathbf{C W S S})_{\mathbf{L}}$ ) if both $E[x(\mathbf{n})]$ (the statistical mean) and $R_{x x}(\mathbf{n}, \mathbf{m})$ (the autocorrelation function) are periodic in $\mathbf{n}$ with periodicity matrix $\mathbf{L}$.

\section{APPENDIX II}

We can compute $((\mathbf{n}))_{M}$ as follows:

$$
((\mathbf{n}))_{\mathbf{M}}=\mathbf{n}-\mathbf{M}\left\lfloor\mathbf{M}^{-1} \mathbf{n}\right\rfloor
$$

where the floor operation is performed on every element of the vector argument [32]. Because $\mathbf{M}^{-1}$ is in general a matrix with rational elements, it is subject to round-off error due to the finite precision of computers. The floor operation $\left[\mathbf{M}^{-1} \mathbf{n}\right\rfloor$ is very sensitive to this noise, so the above approach is not appropriate for computation. The following expression gives an alternative:

$$
((\mathbf{n}))_{\mathbf{M}}=\mathbf{M}([\text { adjugate of } \mathbf{M}] \mathbf{n} \bmod [\operatorname{det} \mathbf{M}]) /[\operatorname{det} \mathbf{M}]
$$

where the modulo operation is performed on every element of [adjugate of $\mathbf{M}$ ] $\mathbf{n}$. This approach is not subject to round-off error because all arithmetic operations are performed on integers.

\section{APPENDIX III}

Let $\mathbf{M}=\mathbf{U} \boldsymbol{\Lambda} \mathbf{V}$ be a Smith form of M.We want to show that for all $\mathbf{b}$ in $\mathscr{N} \mathbf{\Lambda}),((\mathbf{U b}))_{\mathrm{M}}$ are distinct. Suppose there exist $\mathbf{b}_{1}$ and $\mathbf{b}_{2}$ in $\left.\mathscr{N} \mathbf{\Lambda}\right)$ such that $\left(\left(\mathbf{U} \mathbf{b}_{1}\right)\right)_{\mathbf{M}}=\left(\left(\mathbf{U b}_{2}\right)\right)_{\mathbf{M}}$. In other words, there exist two real vectors $x_{1}$ and $x_{2}$ in $[0,1)^{D}$ such that $\left(\left(\mathbf{U} \boldsymbol{\Lambda} \mathbf{x}_{1}\right)\right)_{\mathbf{M}}=\left(\left(\mathbf{U} \boldsymbol{\Lambda} \mathbf{x}_{2}\right)\right)_{\mathbf{M}}$. This implies $\mathbf{U} \Lambda \mathbf{x}_{1}=\mathbf{U} \boldsymbol{\Lambda} \mathbf{x}_{2}+\mathbf{M n} \mathbf{n}_{0}$ where $\mathbf{n}_{0}$ is an integer vector. Let $\mathbf{x}=\mathbf{x}_{1}-\mathbf{x}_{2}$ so $\mathbf{x} \in(-1,1)^{D}$. We then have $\mathbf{x}=\mathbf{V n}_{0}$, which implies that $\mathbf{x}=\mathbf{0}$. We conclude that $\mathbf{b}_{1}$ and $\mathbf{b}_{2}$ are identical, and hence $((\mathbf{U b}))_{M}$ are distinct.

\section{REFERENCES}

[1] R. E. Crochiere and L. R. Rabiner, Multirate Digital Signal Processing. Englewood Cliffs, NJ: Prentice-Hall, 1983.

[2] M. Vetterli, "A theory of multirate filter banks," IEEE Trans. Acous., Speech, Signal Processing, vol. ASSP-35, pp. 356-372, Mar. 1987.

[3] P. P. Vaidyanathan, "Multirate digital filters, filter banks, polyphase networks, and applications: A tutorial," Proc. IEEE, pp. 56-93, Jan. 1990.

[4] R. E. Crochiere, S. A. Webber, and J. L. Flanagan, "Digital coding of speech in subbands," Bell Sys. Tech. J., vol. 55, pp. 1069-1085, Oct. 1976.

[5] N. S. Jayant and P. Noll, Digital Coding of Waveforms. Englewood Cliffs, NJ: Prentice Hall, 1984

[6] R. N. J. Veldhuis, M. Breeuwer, and R. G. van der Waal, "Subband coding of digital audio signals," Philips J. Res., vol. 44, pp. $329-343,1989$.

[7] R. M. Mersereau and T. C. Speake, "The processing of periodically sampled multidimensional signals," IEEE Trans. Acoust., Speech, Signal Processing, vol. 31, no. 1, pp. 188-194, Feb. 1983.

[8] M. Vetterli, "Multidimensional subband coding: Some theory and algorithms," Signal Processing, vol. 6, no. 2, pp. 97-112, Feb. 1984.

[9] E. Dubois, "The sampling and reconstruction of time-varying imagery with application in video systems," Proc. IEEE, vol. 73, pp. 502-522, Apr. 1985.

[10] J. W. Woods and S. D. O'Neil, "Subband coding of images," IEEE Trans. Acoust., Speech, Signal Processing, vol. ASSP-34, no. 5, pp. 1278-1288, Oct. 1986.

[11] R. Ansari and C.-L. Lau, "Two-dimensional IIR filters for exact reconstruction in tree structured subband decomposition," Electron. Lett., vol. 23, pp. 633-634, June 1987.

[12] H. Gharavi and A. Tabatabai, "Subband coding of monochrome and color images," IEEE Trans. Circuits Syst., vol. 35, pp. 207-214, Feb. 1988.

[13] R. Ansari and S. H. Lee, "Two-dimensional non-rectangular interpolation, decimation and filter banks," in Proc. IEEE Int. Conf. ASSP (New York), Apr. 1988.

[14] E. Viscito and J. P. Allebach, "Design of perfect reconstruction multi-dimensional filter banks using cascaded Smith form matrices," in Proc. IEEE Int. Symp. Circuits Syst. (Espoo, Finland), June 1988, pp. 831-834.

[15] V. C. Liu and P. P. Vaidyanathan, "Alias cancellation and distortion elimination in multidimensional QMF banks," Proc. IEEE Int. Symp. Circuits Syst. (Espoo, Finland), June 1988, pp. 1281-1284.

[16] M. Renfors, "Multi-dimensional sampling structure conversion with one-dimensional Nth-band filters," in Proc. IEEE Int. Symp. Circuits Syst. (Portland, OR), May 1989, pp. 1502-1507.

[17] E. P. Simoncelli and E. H. Adelson, "Nonseparable extensions of quadrature mirror filters to multiple dimensions," Proc. IEEE, vol. 78, pp. 652-664, Apr. 1990.

[18] G. Karlsson and M. Vetterli, "Theory of two-dimensional multirate filter banks," IEEE Trans. Acoust., Speech, Signal Processing, vol. 38, no. 6, pp. 925-937, June 1990.

[19] R. Ansari and C. Guillemot, "Exact reconstruction filter banks using diamond FIR filters," Commun., Control, Signal Processing, vol. 2, pp. 1412-1424, July 1990

[20] M. J. T. Smith and S. L. Eddins, "Analysis/Synthesis techniques for subband image coding," IEEE Trans. Acoust., Speech, Signal Processing, vol. 38, no. 8, pp. 1446-1456, Aug. 1990.

[21] M. Vetterli, J. Kovačević, and D. J. Legall, "Perfect reconstruction filter banks for HDTV representation and coding," Signal Processing: Image Commun., vol. 2, no. 3, pp. 349-363, Oct. 1990.

[22] R. H. Bamberger, "The directional filter bank: A multirate filter bank for the directional decomposition of images," Ph.D. dissertation, Georgia Institute of Technology, Atlanta, 1990.

[23] P. P. Vaidyanathan, "Fundamentals of multidimensional multirate digital signal processing," Sadhana, vol. 15, pp. 157-176, Nov. 1990. 
[24] K. R. Rao and P. Yip, Discrete Cosine Transform: Algonithms, Advantages, Applications. New York: Academic, 1990.

[25] T. Chen and P. P. Vaidyanathan, "Multidimensional multirate filters derived from one dimensional filters," Electron. Lett., vol 27, no. 3, pp. 225-228, Jan. 1991.

[26] E. Viscito, and J. P. Allebach, "The analysis and design of multidimensional FIR perfect reconstruction filter banks for arbitrary sampling lattices," IEEE Trans. Circuits Syst., vol. 38, no. 1, pp 29-41, Jan. 1991.

[27] J. Kovačević and M. Vetterli, "The commutativity of up/downsampling in two dimensions," IEEE Trans. on Inform. Theony, vol. 37, no. 3, pp. 695-698, May 1991.

[28] P. P. Vaidyanathan, "The role of Smith-form decomposition of integer-matrices, in multidimensional multirate systems," in Proc IEEE Int. Conf. ASSP (Toronto, Canada), May 1991, pp. 1777 1780 .

[29] - "New results in multidimensional multirate systems," in Proc. IEEE Int. Symp. Circuits Syst. (Singapore), June 1991, pp. 468-471.

[30] J. W. Woods, Subband Image Coding. Norwell, MA: Kluwer, 1991.

[31] T. Chen and P. P. Vaidyanathan, "Commutativity of D-dimensional decimation and expansion matrices, and application to rational decimation systems," in Proc. IEEE Int. Conf. ASSP, vol. 4 (San Francisco), Mar. 1992, pp. 637-640.

[32] B. L. Evans, J. H. McClellan, and R. H. Bamberger, "A symbolic algebra for linear multidimensional multirate systems," in Proc. 1992 Conf. Inform. Sci. Syst. (Princeton, NJ), Mar. 1992.

[33] A. A. C. M. Kalker, "Commutativity of up/down sampling," Elec tron. Lett., vol. 28, no. 6, pp. 567-569, Mar. 1992.

[34] J. Kovačević and M. Vetterli, "Nonseparable multidimensional perfect reconstruction filter banks and wavelet bases for $\mathscr{R}^{n}$," IEEE Trans. on Information Theory, vol. 38, no. 2, pp. 533-555, March 1992.

[35] R. H. Bamberger and M. J. T. Smith, "A filter bank for the directional decomposition of images: Theory and design," IEEE Trans. Signal Processing, vol. 40, no. 4, pp. 882-893, Apr. 1992.

[36] T. Chen and P. P. Vaidyanathan, "Least common right/left multiples of integer matrices and applications to multidimensional multirate systems," in Proc. IEEE Int. Symp. Circuits Syst. (San Diego) May 1992, pp. 935-938.

[37] I. A. Shah and A. A. C. Kalker, "Generalized theory of multidimensional $M$-band filter bank design," in Proc. EUSIPCO-92, Aug. 1992, pp. 969-972.

[38] H. S. Malvar, Signal Processing with Lapped Transforms. Norwood, MA: Artech House, 1992.

[39] T. Chen and P. P. Vaidyanathan, "The role of integer matrices in multidimensional multirate systems," IEEE Trans. Signal Processing, vol. 41, no. 3, Mar. 1993.

[40] P. P. Vaidyanathan, Multirate Systems and Filter Banks, Englewood Cliffs, NJ: Prentice-Hall, 1993.

[41] T. Chen and P. P. Vaidyanathan, "Multidimensional multirate filters and filter banks derived from one dimensional filters," IEEE Trans. Signal Processing, vol. 41, no. 5, May 1993.

[42] B. L. Evans, R. H. Bamberger, and J. H. McClellan, "Rules for multidimensional multirate structures," submitted to IEEE Trans. Signal Processing.

[43] R. A. Gopinath and C. S. Burrus, "On upsampling, downsampling and rational sampling rate filter banks," submitted to IEEE Trans. Signal Processing.

[44] M. Kunt, "Recent HDTV systems," in Signal Processing VI: Theo ries and Applications. New York: Elsevier, 1992, pp. 83-89.

[45] J. W. S. Cassels, An Introduction to the Geonzetry of Numbers. Berlin: Springer-Verlag, 1959.

[46] M. Newman, Integral Matrices. New York: Academic, 1972.

[47] D. P. Petersen and D. Middleton, "Sampling and reconstruction of wave-number-limited functions in $N$-dimersional Euclidean spaces," Information and Control, vol. 5, pp. 279--323, 1962.

[48] S. Mallat, "A theory for multiresolution signal decomposition: the wavelet representation," IEEE Trans. Pattern Anal. Machine Intell. vol. 11 , pp. 674-693, July 1989.

[49] D. E. Dudgeon and R. M. Mersereau, Multidimensional Digital Signal Processing. Englewood Cliffs, NJ: Prentice-Hall, 1984.

[50] R. Ansari, "Efficient IIR and FIR fan filters," IEEE Trans. Circuits Syst., vol. CAS-34, no. 8, pp. 941-945, Aug. 1987

[51] P. P. Vaidyanathan and S. K. Mitra, "Polyphase networks, block digital filtering, LPTV systems, and alias-free QMF banks: A unified approach based on pseudocirculants," IEEE Trans. Acoust., Speech, Signal Processing, vol. 36, no. 3, pp. 381-391, Mar. 1988.

[52] P. P. Vaidyanathan, "Theory and design of $M$-channel maximally decimated quadrature mirror filters with arbitrary $M$, having the perfect-reconstruction property," IEEE Trans. Acoust., Speech, Signal Processing, vol. ASSP-35, no. 4, pp. 476-492, Apr. 1987.

[53] H. J. S. Smith, "On systems of linear indeterminate equations and congruences," Phil. Trans. Roy. Soc. London, vol. 151, pp. 293-326, 1861.

[54] C.-C. Hsiao, "Polyphase filter matrix for rational sampling rate conversions," Proc. IEEE Int. Conf. ASSP (Dallas, TX), Apr. 1987, pp. 2173-2176.

[55] T. Q. Nguyen and P. P. Vaidyanathan, "Maximally decimated perfect reconstruction FIR filter banks with pairwise mirror-image analysis (and synthesis) frequency responses," IEEE Trans. Acoust., Speech, Signal Processing, vol. 36, no. 5, pp. 693-705, May 1988.

[56] V. Sathe and P. P. Vaidyanathan, "Effects of multirate systems on the statistical properties of random inputs," IEEE Trans. Signal Processing, vol. 41, no. 1, pp. 131-146, Jan. 1993.

[57] F. R. Gantmacher, The Theory of Matrices, vol. 1. New York: Chelsea Publishing Co. 1977.

[58] T. Kailath, Linear Systems. Englewood Cliffs, NJ: Prentice-Hall, 1980.

[59] M. Vidyasagar, Control System Synthesis: A Factorization Approach. Cambridge, MA: M.I.T. Press, 1985.

[60] C. C. MacDuffee, The Theory of Matrices. New York: Chelsea Publishing Co., 1946.

[61] G. David Forney, Jr., "Convolutional codes I: Algebraic structure," IEEE Trans. Inform. Theory, vol. IT-16, no. 6, pp. 720-738, Nov. 1970.

[62] Y. Neuvo, C. Y. Dong, and S. K. Mitra, "Interpolated finite impulse response filters," IEEE Trans. Acoust., Speech, Signal Processing, vol. ASSP-32, no. 3, pp. 563-570, June 1984.

[63] L. R. Rabiner and B. Gold, Theory and Application of Digital Signal Processing. Englewood Cliffs, NJ: Prentice-Hall, 1975.

[64] A. Guessoum and R. M. Mersereau, "Fast algorithms for the multidimensional discrete Fourier transform," IEEE Trans. Acoust., Speech, Signal Processing, vol. ASSP-34, no. 4, pp. 937-943, Aug. 1986.

[65] R. E. Blahut, Theory and Practice of Error Control Codes. Reading, Massachusetts: Addison-Wesley, 1983.

[66] J. A. Sjogren (AFOSR), private communication.

[67] T. Chen and P. P. Vaidyanathan, "On the choice of rational decimation systems for multidimensional signals," in Proc. IEEE Int. Conf. ASSP (Minneapolis, MN), Apr. 1993.

[68] R. Manduchi, G. M. Cortelazzo, and G. A. Mian, "Multistage sampling structure conversion of video signals," preprint.

\section{SELECTED REFERENCES BY TOPIC}

Lattices and Integer Matrices

[53] H. J. S. Smith, 1861

[60] C. C. MacDuffee, 1946.

[45] J. W. S. Cassels, 1959.

[46] M. Newman, 1972.

[59] M. Vidyasagar, 1985.

One-Dimensional Multirate Systems

[1] R. E. Crochiere and L. R. Rabiner, 1983.

[2] M. Vetterli, 1987.

[54] C.-C. Hsiao, 1987.

[55] T. Q. Nguyen and P. P. Vaidyanathan, 1988.

[3] P. P. Vaidyanathan, 1990.

[56] V. Sathe and P. P. Vaidyanathan, 1993.

Multidimensional Lattice Sampling

[47] D. P. Petersen and D. Middleton, 1962.

[7] R. M. Mersereau and T. C. Speake, 1983.

[49] D. E. Dudgeon and R. M. Mersereau, 1984.

[9] E. Dubois, 1985.

Multidimensional Multirate Theory

[7] R. M. Mersereau and T. C. Speake, 1983.

[8] M. Vetterli, 1984.

[15] V. C. Liu and P. P. Vaidyanathan, 1988. 
[18] G. Karlsson and M. Vetterli, 1990.

[26] E. Viscito and J. P. Allebach, 1991

[27] J. Kovačević and M. Vetterli, 1991.

[31] T. Chen and P. P. Vaidyanathan, 1992

[32] B. L. Evans, J. H. McClellan, and R. H. Barnberger, 1992.

[33] A. A. C. M. Kalker, 1992.

[34] J. Kovačević and M. Vetterli, 1992

[39] T. Chen and P. P. Vaidyanathan, 1993.

[67] T. Chen and P. P. Vaidyanathan, 1993

[43] R. A. Gopinath and C. S. Burrus, submitted for publication.

\section{Multidimensional Systems and Filter Design}

[64] A. Guessoum and R. M. Mersereau, 1986.

[11] R. Ansari and C.-L. Lau, 1987.

[50] R. Ansari, 1987.

[13] R. Ansari and S. H. Lee, 1988.

[14] E. Viscito and J. P. Allebach, 1988.

[16] M. Renfors, 1989.

[19] R. Ansari and C. Guillemot, 1990

[20] M. J. T. Smith and S. L. Eddins, 1990.

[26] E. Viscito and J. P. Allebach, 1991.

[25] T. Chen and P. P. Vaidyanathan, 1991.

[35] R. H. Bamberger and M. J. T. Smith, 1992.

[37] I. A. Shah and A. A. C. Kalker, 1992.

[41] T. Chen and P. P. Vaidyanathan, 1993.

\section{Image and Video Coding}

[8] M. Vetterli, 1984.

[5] N. S. Jayant and P. Noll, 1984

[10] J. W. Woods and S. D. O'Neil, 1986

[12] H. Gharavi and A. Tabatabai, 1988.

[20] M. J. T. Smith and S. L. Eddins, 1990.

[30] J. W. Woods, 1991.

[44] M. Kunt, 1992.

Books and Papers of Tutorial Value

[7] R. M. Mersereau and T. C. Speake, 1983.

[1] R. E. Crochiere and L. R. Rabiner, 1983.

[49] D. E. Dudgeon and R. M. Mersereau, 1984.

[9] E. Dubois, 1985.

[2] M. Vetterli, 1987

[3] P. P. Vaidyanathan, 1990.

[23] P. P. Vaidyanathan, 1990

[26] E. Viscito and J. P. Allebach, 1991

[30] J. W. Woods, 1991.

[44] M. Kunt, 1992.

[40] P. P. Vaidyanathan, 1993.

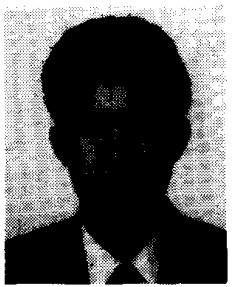

Tsuhan Chen (S'90) was born in Taipei, Taiwan, Republic of China, on November 7, 1965. He received the B.S. degree in electrical engineering from the National Taiwan University, Taipei, Taiwan, in 1987, the M.S. degree in electrical engineering from the California Institute of Technology, Pasadena, in 1990, and is currently working toward the $\mathrm{Ph} . \mathrm{D}$. degree in electrical engineering at the California Institute of Technology.

His main research interests are in digital signal processing, multirate filter banks, and multidimensional multirate systems.

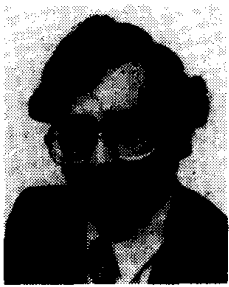

P. P. Vaidyanathan (S'80-M'83-SM'88-F'91) was borm in Calcutta, India, on October 16, 1954. He received the B.Sc. (Hons.) degree in physics and the B.Tech. and M.Tech. degrees in radiophysics and electronics, all from the University of Calcutta, India, in 1974, 1977, and 1979, respectively, and the Ph.D. degree in electrical and computer engineering from the University of California, Santa Barbara, in 1982

He was a postdoctoral fellow at the University of California, Santa Barbara, from September 1982 to March 1983. In March 1983 he joined the electrical engineering department of the California Institute of Technology, Pasadena, as an Assistant Professor, and since 1988 has been an Associate Professor of electrical engineering there. His main research interests are in digital signal processing, multirate systems, wavelet transforms, and adaptive filtering.

Dr. Vaidyanathan served as Vice-Chairman of the Technical Program committee for the 1983 IEEE International Symposium on Circuits and Systems and as an Associate editor for the IEEE TrANSACTIONS ON CIRCUITS AND SYSTEMS for 1985-1987. He also served as the Technical Program Chairman for the 1992 IEEE International Symposium on Circuits and Systems. He was a recipient of the Award for Excellence in Teaching at the California Institute of Technology for 1983-1984. He also received the NSF's Presidential Young Investigator award in 1986 In 1989 he received the IEEE ASSP Senior Award for his paper on multirate perfect-reconstruction filter banks. In 1990 he was the recipient of the S. K. Mitra Memorial Award from the Institute of Electronics and Telecommuncations Engineers, India, for his joint paper in the IETE journal. 